Preprint typeset in JHEP style - HYPER VERSION

\title{
Cosmological perturbations in a family of deformations of general relativity
}

\author{
Kirill Krasnov \\ School of Mathematical Sciences, University of Nottingham, Nottingham NG7 2RD, UK \\ E-mail: kirill.krasnov@nottingham.ac.uk \\ Yuri Shtanov \\ Bogolyubov Institute for Theoretical Physics, Kiev 03680, Ukraine \\ Faculty of Physics, Taras Shevchenko National University of Kiev, Ukraine \\ E-mail: shtanov@bitp.kiev.ua
}

\begin{abstract}
We study linear cosmological perturbations in a previously introduced family of deformations of general relativity characterized by the absence of new degrees of freedom. The homogeneous and isotropic background in this class of theories is unmodified and is described by the usual Friedmann equations. The theory of cosmological perturbations is modified and the relevant deformation parameter has the dimension of length. Gravitational perturbations of the scalar type can be described by a certain relativistic potential related to the matter perturbations just as in general relativity. A system of differential equations describing the evolution of this potential and of the stress-energy density perturbations is obtained. We find that the evolution of scalar perturbations proceeds with a modified effective time-dependent speed of sound, which, contrary to the case of general relativity, does not vanish even at the matter-dominated stage. In a broad range of values of the length parameter controlling the deformation, a specific transition from the regime of modified gravity to the regime of general relativity in the evolution of scalar perturbations takes place during the radiation domination. In this case, the resulting power spectrum of perturbations in radiation and dark matter is suppressed on the comoving spatial scales that enter the Hubble radius before this transition. We estimate the bounds on the deformation parameter for which this suppression does not lead to observable consequences. Evolution of scalar perturbations at the inflationary stage is modified but very slightly and the primordial spectrum generated during inflation is not noticeably different from the one obtained in general relativity.
\end{abstract}

KEYWORDS: cosmological perturbation theory, modified gravity. 


\section{Contents}

1. Introduction 2

2. A family of deformations of GR

2.1 Preliminaries: two-form field and the metric

2.2 The gravitational action 6

2.3 Coupling to matter 0

2.4 Field equations 8

2.5 Large-distance modifications 10

2.6 The cosmological constant and vacuum energy 11

3. Homogeneous and isotropic universe 11

4. Linearized field equations 12

5. Classification of perturbations 14

5.1 Scalar sector 15

5.2 Vector sector 16

5.3 Tensor sector 16

6. Scalar perturbations 16

6.1 Curvature 16

6.2 Field equations 17

6.3 Conservation equations 18

6.4 Equations for the "non-metricity" scalar $\chi \quad 18$

6.5 New relativistic potential 20

6.6 Applicability limits of the theory 21

7. Evolution of scalar perturbations 21

7.1 Inflation 21

7.2 Evolution with a generic equation of state 24

7.3 Radiation domination 26

7.4 Matter domination 28

7.5 Lambda domination 29

8. Effects of the modification of gravity on the power spectrum 30

9. Discussion 36

A. Derivation of equations describing the radiation-dark-matter system 38 
B. Tensor sector 39

B.1 Curvatures 39

B.2 Field equations 40

B.3 Action principle and Hamiltonian analysis 40

\section{Introduction}

The future astrophysical and cosmological data will, among other things, allow one to perform deeper tests of our theory of gravity, general relativity (GR). Although there are no compelling reasons to question seriously the validity of GR, several puzzles associated with its long-distance behavior, primarily those connected with the phenomena of dark energy and dark matter, make it an important task to see whether GR continues to be as good a description of gravity on astrophysical and cosmological scales as it is on the scales of Solar System. Modern literature is abundant with various proposals of modified gravity addressing this issue. A common feature of almost all such modifications is that new propagating degrees of freedom are introduced in the gravitational sector, in the form of additional fields or higher derivatives of the metric field. A typical example is $f(R)$ gravity, which in its original variables is a purely metric theory with higher derivatives but also can be transformed into a scalar-tensor theory. Extensions of this kind typically change both the Friedmann equations describing the evolution of a homogeneous and isotropic universe and the equations describing cosmological perturbations, by which modifications they can be tested.

It is, perhaps, less known that one can modify GR without introducing any new propagating degrees of freedom. Modification of this kind, in particular, should be expected not to change the homogeneous background evolution in cosmology, affecting only the way the perturbations evolve. Some of the generic features of this scheme were explored in [1] without specifying a concrete theory.

A specific class of modifications of GR without new propagating degrees of freedom was proposed by one of us in [2], with the same class of theories envisaged earlier in a different formulation in [3]. These theories legitimately can be called deformations of GR in the sense that there exist continuous paths in the parameter space smoothly connecting any of them with GR. The theories in this class provide a new framework for testing general relativity, since, using the observational data, one can determine how close is the real-world gravity to GR within a class of its deformations. In one of our previous papers [4], we were concerned with the spherically symmetric solution in this class of theories, its features and possible implications. In the present paper, we turn to the description of cosmology. Our aim is to set a stage for possible tests of the theories of [2] by working out the modified equations describing the evolution of cosmological perturbations.

Before describing the class of theories under investigation in detail, it is worth discussing their basic properties in elementary terms. If viewed as purely metric theories, 
they can be regarded as the usual effective theories with a nonlocal Lagrangian that can be expanded into an infinite series of local terms built from the curvature invariants: $\mathcal{L}=\alpha_{1} R+\alpha_{2} R^{2}+\alpha_{3} R_{\mu \nu} R^{\mu \nu}+\alpha_{4} R_{\mu \nu \sigma \tau} R^{\mu \nu \sigma \tau}+\ldots$ The coefficients in front of these invariants, however, are not arbitrary in the present case, but are such that the whole infinite series can be resummed with the help of auxiliary non-propagating fields. In the end, one obtains a local second-derivative theory with only two propagating degrees of freedom (two polarizations of the gravitational wave), and a certain limit can be taken to recover GR if one so wishes, see [0].

To elucidate this idea, we illustrate it on a simple example of a single field with a higher-derivative action. Let us start from the linearized Lagrangian

$$
\mathcal{L}^{(2)}[h]=\frac{1}{2} h \square h,
$$

where $\square=\nabla_{\mu} \nabla^{\mu}$ and our signature convention is $(-,+,+,+)$. One can think of the field $h$ as that of the graviton with the factor of the inverse Newton's constant $G^{-1}$ absorbed into the definition of $h$ to endow it with the canonical mass dimension $[h]=1$. All tensor indices of $h$ have been discarded in order to make the discussion as simple as possible.

Lagrangian (1.1) can be modified by introducing higher-derivative non-renormalizable terms. For any finite number of such terms, the resulting theory has additional propagating modes corresponding to the poles of the arising propagator. However, when an infinite number of such higher-derivative terms is present, they sometimes can be resummed into a denominator so that the only pole of the arising propagator is still that of the massless graviton. One of the simplest examples realizing this idea is

$$
\mathcal{L}[h]=\frac{1}{2} h\left(\square+\ell^{2} \square^{2}+\ell^{4} \square^{3}+\ell^{6} \square^{4}+\ldots\right) h=\frac{1}{2} h \frac{\square}{1-\ell^{2} \square} h .
$$

Here, $\ell$ is the length scale of the modification. It is clear that the usual "graviton" is the only propagating mode in (1.2). To see this explicitly, note that the non-local theory (1.2) can be obtained from the local one

$$
\mathcal{L}[h, \chi]=\frac{1}{2} \chi\left(\square-m^{2}\right) \chi+\chi \square h+\frac{1}{2} h \square h,
$$

where $m=\ell^{-1}$, by integrating out the auxiliary field $\chi$. Indeed, the equation for $\chi$ that stems from (1.3) is formally solved by

$$
\chi=\frac{\square h}{m^{2}-\square} .
$$

Substituting this into (1.3), one obtains (1.2). At the level of action (1.3), the limit $m \rightarrow \infty$ that gives back (1.1) works by making the field $\chi$ infinitely massive, and thus effectively decoupling it from the theory. At the same time, writing action (1.3) in terms of the new field variable $\tilde{h}=h+\chi$,

$$
\mathcal{L}[\tilde{h}, \chi]=\frac{1}{2} \tilde{h} \square \tilde{h}-\frac{1}{2} m^{2} \chi^{2},
$$

one can see that the field $\chi$ is an auxiliary non-propagating field, and the theory (1.3) has the same number of degrees of freedom and structure as the one we started from, Eq. (1.1). 
Thus, the described modification (1.2) is just a field redefinition, which gives another way to understand why no new propagating degrees of freedom is introduced in this case. ${ }^{1}$

The described elementary modification scheme is (almost) identical to the one at play in the class of theories [2] at the linearized level. However, in theories [2], it is extended to a full non-linear level in a non-trivial fashion. It then turns out that, by the same trick of introducing auxiliary non-propagating fields, quite non-trivial terms in the arising effective metric Lagrangian can be reproduced, for instance, the Riemann ${ }^{3}$ term of importance in quantum gravity at two loops; see [5].

To summarize, the idea of the deformations of GR [2] is that higher-derivative terms in the effective metric Lagrangian can be added without introducing extra degrees of freedom, and the theory is then made local at the expense of introducing some non-propagating auxiliary fields. In the class of theories [2], this idea is realized elegantly by combining the metric and auxiliary fields into a single field - an SU(2) Lie-algebra-valued two-form.

Another general comment should be made about the class of theories under investigation in this paper. The above discussion seemingly implies that they can be regarded as ultraviolet modifications of general relativity. Indeed, usually, modifications generated by the addition of higher-derivative terms to the Hilbert-Einstein Lagrangian are of relevance at high energies, e.g., close to the Planck scale, but are unimportant at low energies of relevance in cosmology. However, our above example shows that this interpretation may not always be valid. Indeed, the theory (1.2) has the field and dynamical content equivalent to that of (1.1). This property will be lost after truncation of the series in (1.2) or after the violation of the delicate relations between the coefficients in this infinite series. Through these relations, the high-derivative terms (potentially important in the ultraviolet) are all connected with the low-derivative terms (important in the infrared). In the case of the modified gravity theory to be considered, this UV/IR interplay will be seen in the fact that the theory can be generated from the self-dual formulation of general relativity simply by making the cosmological constant a function of the variable which in GR has the meaning of the Weyl curvature (more on this below). This allows gravity to be modified on cosmological scales rather naturally, simply by making the usual cosmological constant a slowly varying function of the curvature. At the same time, it is easy to remain consistent with GR on Solar-System scales by requiring the "cosmological function" to be approximately constant in the relevant region of curvatures (see the next section for more details). In this sense, the theory simultaneously looks like an infrared modification of gravity. This interplay between the ultraviolet and infrared modification is an interesting feature that characterizes the class of theories under investigation.

The plan of the paper is as follows. In Sec. 2, we briefly describe the family of deformations of GR which is the subject of this study. In Sec. 3, we describe the homogeneous isotropic Universe and the usual Friedmann equations in the language of two-forms adopted in this paper. Section 4 describes the linearized field equations around the evolving cos-

\footnotetext{
${ }^{1}$ One cannot discard the field $\chi$ in $(\sqrt{1.5})$ if the "matter" part of the action interacts with the original field $h \equiv \tilde{h}-\chi$. In this case, there arises a theory with non-propagating auxiliary scalar $\chi$ that couples to matter, resembling the modified source gravity [6]. This will also be the case in the modified-gravity theory under consideration in this paper.
} 
mological background. In Sec. 5, we classify the types of perturbations and describe the physical gauge-invariant quantities. Section 6 obtains equations describing perturbations of the scalar type. These are analyzed in details in Sec. 7. We study the effect of modification on the CMB and matter power spectrum in Sec. 8. We conclude with a brief discussion of the results obtained. Preliminary results concerning tensor modes are described in Appendix B.

\section{A family of deformations of GR}

In this section, we briefly review the theory under investigation in this work. For more details, the reader is directed to a recent description in [5].

\subsection{Preliminaries: two-form field and the metric}

As we mentioned in the introduction, in our theory the metric and the auxiliary nonpropagating fields are "unified" into a single two-form field $B_{\mu \nu}^{i}, i=1,2,3$, where $\mu, \nu$ are spacetime indices. The (complexified) rotation group $\mathrm{SO}(3, \mathbb{C})$ acts on the two-form $B_{\mu \nu}^{i}$ via $B_{\mu \nu}^{i} \rightarrow O_{j}^{i} B_{\mu \nu}^{j}, O_{j}^{i} \in \mathrm{SO}(3, \mathbb{C})$, and the theory is invariant under the corresponding gauge transformations as well as under the action of spacetime diffeomorphisms.

The physical metric of the spacetime, to which all fields are supposed to couple universally, is determined uniquely by the two-form field. To begin with, we require that the triple of two-forms $B_{\mu \nu}^{i}$ be self-dual with respect to this metric, which determines its conformal class. In other words, introducing the associated volume form $\epsilon_{\mu \nu \rho \sigma}$ for a metric $g_{\mu \nu}$ and imposing the self-duality conditions ${ }^{2}$

$$
\frac{1}{2} \epsilon_{\mu \nu}^{\rho \sigma} B_{\rho \sigma}^{i}=\mathrm{i} B_{\mu \nu}^{i}
$$

fixes the metric $g_{\mu \nu}$ modulo a conformal rescaling.

An equivalent explicit description of the conformal class of metrics determined by $B_{\mu \nu}^{i}$ is given by the formula

$$
\sqrt{-\operatorname{det}\left(g_{\mu \nu}\right)} g_{\mu \nu} \propto \tilde{\epsilon}^{\alpha \beta \gamma \delta} B_{\mu \alpha}^{i} B_{\nu \beta}^{j} B_{\gamma \delta}^{k} \epsilon^{i j k}
$$

where $\tilde{\epsilon}^{\alpha \beta \gamma \delta}$ is a densitized completely anti-symmetric tensor having components \pm 1 in any coordinate system, and the proportionality symbol is used here to denote that the metric is defined up to conformal rescalings. The reality conditions imposed on the theory are that the conformal metric (2.2) is real Lorentzian. To fix a unique metric from its conformal class, it suffices to specify its volume form. Below, we shall explain how the triple $B_{\mu \nu}^{i}$ defines not only a conformal class of metrics, but a volume form as well.

Vice versa, given a real metric $g_{\mu \nu}$ with Lorentzian signature, one can introduce the corresponding tetrad one-forms $\theta_{\mu}^{I}, I=0,1,2,3$, so that the line element reads

$$
d s^{2}=\theta^{I} \otimes \theta^{J} \eta_{I J}
$$

\footnotetext{
${ }^{2}$ The spacetime indices in (2.1) are raised with the use of the inverse metric $g^{\mu \nu}$.
} 
where $\eta_{I J}$ is the Minkowski metric. Then, introducing an arbitrary time plus space split $I=(0, a)$ of the "internal" index $I$, we can define a set of self-dual metric two-forms $\Sigma^{a}$ :

$$
\Sigma^{a}=\mathrm{i} \theta^{0} \wedge \theta^{a}-\frac{1}{2} \epsilon^{a b c} \theta^{b} \wedge \theta^{c}
$$

Here, the letters $a, b, c$ are new $\mathrm{SO}(3)$ indices. It is convenient to distinguish between the original $\mathrm{SO}(3)$ indices in $B^{i}$ and the new index that appears in the metric two-forms $\Sigma^{a}$, which justifies the notation. Let us also give an expression for a convenient basis in the space of anti-self-dual two-forms:

$$
\bar{\Sigma}^{a}=\mathrm{i} \theta^{0} \wedge \theta^{a}+\frac{1}{2} \epsilon^{a b c} \theta^{b} \wedge \theta^{c}
$$

Any other set of self-dual two-forms can be decomposed in the basis provided by $\Sigma^{a}$. Thus, we can write

$$
B^{i}=b_{a}^{i} \Sigma^{a}
$$

Therefore, the main dynamical field of the theory can always be chosen as a collection of a metric $g_{\mu \nu}$ that, in turn, defines the metric forms $\Sigma^{a}$, together with the nine scalars forming the matrix $b_{a}^{i}$. These scalars will later be seen to be non-dynamical and determined by other fields present in the system. We do not impose any independent reality conditions on $b_{a}^{i}$ as such reality conditions are induced once $b_{a}^{i}$ are determined in terms of other fields. Thus, $b_{a}^{i}$ are in general complex fields. Note that, as the metric $g_{\mu \nu}$ undergoes a conformal transformation $g_{\mu \nu} \rightarrow \Omega^{2} g_{\mu \nu}$, the metric self-dual two-forms transform as $\Sigma^{a} \rightarrow \Omega^{2} \Sigma^{a}$. Thus, if the scalars $b_{a}^{i}$ transform as $b_{a}^{i} \rightarrow \Omega^{-2} b_{a}^{i}$, then the original two-forms $B^{i}$ are unchanged. A certain normalization condition on the scalars $b_{a}^{i}$ will be introduced below to fix this conformal freedom, thus fixing a particular metric from the conformal class of (2.2).

\subsection{The gravitational action}

The vacuum theory of gravity under consideration is described by the following action:

$$
S[B, A]=\frac{\mathrm{i}}{8 \pi G} \int\left[B^{i} \wedge F^{i}(A)-\frac{1}{2} V\left(B^{i} \wedge B^{j}\right)\right] .
$$

Here, $A^{i}$ is an $\mathrm{SO}(3, \mathbb{C})$ connection field, $F^{i}(A)=d A^{i}+(1 / 2) \epsilon^{i j k} A^{j} \wedge A^{k}$ is its curvature, and $V\left(M^{i j}\right)$ is a (holomorphic) function of a complex symmetric $3 \times 3$ matrix variable $M^{i j}$. It is required to be a homogeneous function of degree one: $V\left(\alpha M^{i j}\right)=\alpha V\left(M^{i j}\right)$ for any number $\alpha$, so that it can be applied to a matrix-valued $B^{i} \wedge B^{j}$ four-form, with the result being again a four-form; see [5] for more details. The function $V\left(M^{i j}\right)$ is also required to be a scalar with respect to the $\mathrm{SO}(3, \mathbb{C})$ gauge transformations. In action (2.7), this function plays the role of potential for the $B^{i}$ field.

Using representation (2.6), let us introduce the "internal metric"

$$
h^{i j}:=b_{a}^{i} b_{b}^{j} \delta^{a b}
$$


which is a symmetric $3 \times 3$ matrix, in general complex. It turns out to be useful to parameterize it by its trace $h=h^{i j} \delta_{i j}$ and trace-free part $H^{i j}$ :

$$
h^{i j}=\frac{1}{3} h\left(\delta^{i j}+H^{i j}\right) .
$$

Then the potential $V\left(B^{i} \wedge B^{j}\right)$ gives rise to the $\mathrm{SO}(3, \mathbb{C})$-invariant function $V\left(h^{i j}\right)$, which can be presented as follows:

$$
V\left(h^{i j}\right)=\frac{1}{3} h U(H), \quad U(H)=\Lambda_{0}-\frac{1}{8 \ell^{2}} \operatorname{Tr} H^{2}+\mathcal{O}\left(H^{3}\right) .
$$

Here, $U(H)$ is a function of the traceless matrix $H^{i j}$ with dimensions of curvature, and we have expanded it in powers of $H^{i j}$ assuming that this potential function is analytic in the neighborhood of $H^{i j}=0$. Below we will see that the case of $h^{i j}=\delta^{i j}$ corresponds to general relativity, so that the traceless matrix $H^{i j}$ parameterizes the deformation away from GR. The constant term $\Lambda_{0}$ in decomposition (2.10) will play the role of the cosmological constant. The quantity $\ell$ is a new parameter of the theory with dimensions of length. In principle, in the theory under consideration, there is scope for making this parameter complex, but, in this paper, we shall only consider the simplest case of real $\ell$. The numerical constant in the expansion in (2.10) is introduced for future convenience. We will see that only negative sign of the second term in (2.10) describes a theory without instabilities, which explains our choice of this sign.

\subsection{Coupling to matter}

As we have stated in Sec. 2.1, the physical metric which universally couples to matter belongs to the conformal class determined by the two-form field $B^{i}$. It is necessary to fix the remaining conformal freedom and to specify this metric uniquely.

Under conformal transformations, described in the end of Sec. 2.1, the "internal metric" introduced in (2.8) transforms as $h^{i j} \rightarrow \Omega^{-4} h^{i j}$. One can specify the physical metric by fixing the conformal ambiguity for $h^{i j}$. To do this, we introduce another scalar (holomorphic) function $R\left(h^{i j}\right)$ of the matrix $h^{i j}$ that is required to be homogeneous of degree one in the components of $h^{i j}: R\left(\alpha h^{i j}\right)=\alpha R\left(h^{i j}\right)$. The conformal freedom of $h^{i j}$ is then fixed by the condition

$$
R\left(h^{i j}\right)=1 .
$$

While this prescription for fixing the physical metric may seem arbitrary at first, it can be shown to arise quite naturally by considering the motion of a "small body" in the theory described by (2.7); see [7].

We normalize the function $R\left(h^{i j}\right)$ so that $R\left(\delta^{i j}\right)=1$. The potential $R\left(h^{i j}\right)$ can then be decomposed similarly to 2.10$)$ :

$$
R(h)=\frac{1}{3} h U_{m}(H), \quad U_{m}(H)=1-\frac{g}{2} \operatorname{Tr} H^{2}+\mathcal{O}\left(H^{3}\right),
$$

where $U_{m}(H)$ is a new function of the traceless matrix $H \equiv H^{i j}$, again assumed to be analytic in the neighborhood of $H^{i j}=0$. The quantity $g$ is a dimensionless parameter. 
Just as with the case of $\ell$, in the theory under consideration, there is scope for making the parameter $g$ complex. However, in this paper, we shall only consider the simplest case of real $\ell$ and $g$. Note that, once the condition (2.11) is imposed, one can use it to express the trace part of the matrix $h^{i j}$ in terms of its traceless part.

We shall see that only the terms written in (2.10) and (2.12) are going to matter for the linearized theory we are going to consider. Thus, the only new parameters that we will have to consider on top of those available in GR are the length parameter $\ell$ in $(2.10)$ and the dimensionless constant $g$ in 2.12) that can be of any sign.

In the class of theories under consideration, matter fields will in general couple to the two-form $B^{i}$ that plays the role of the fundamental geometrical field of the theory. However, for the purposes of this paper, we assume that our matter content is "standard" in the sense that our material components couple to the spacetime metric in the conformal class defined by $B^{i}$ and further selected by the condition (2.11). Thus, we assume that the dependence of the matter action $S_{m}$ on $B^{i}$ is such that $S_{m}[B]$ is a functional of $g_{\mu \nu}$ and depends on the scalars $b_{a}^{i}$ solely via the function $R(h)$. This allows us to write:

$$
\delta S_{m}=\int \sqrt{-\operatorname{det}\left(g_{\mu \nu}\right)}\left(T_{\mu \nu} \delta g^{\mu \nu}+\frac{\partial S_{m}}{\partial R} \frac{\partial R}{\partial b_{a}^{i}} \delta b_{a}^{i}\right) .
$$

However, since the two-form field $B^{i}$ does not transform under the conformal transformations $\delta g_{\mu \nu}=\varepsilon g_{\mu \nu}, \delta b_{a}^{i}=-\varepsilon b_{a}^{i}$, any material action that arises from $S_{m}[B]$ is also conformally-invariant. This immediately gives:

$$
T=\frac{\partial S_{m}}{\partial R} \frac{\partial R}{\partial b_{a}^{i}} b_{a}^{i}
$$

where $T:=T_{\mu \nu} g^{\mu \nu}$, or, using the homogeneity of $R(h)$ :

$$
\frac{\partial S_{m}}{\partial R}=\frac{T}{2 R} .
$$

In other words, the variation of the material action contains the following two terms:

$$
\delta S_{m}=\int \sqrt{-\operatorname{det}\left(g_{\mu \nu}\right)}\left(T_{\mu \nu} \delta g^{\mu \nu}+\frac{T}{2 R} \frac{\partial R}{\partial b_{a}^{i}} \delta b_{a}^{i}\right) .
$$

After the condition (2.11) is imposed, the quantity $T_{\mu \nu}$ becomes the usual stress-energy tensor of matter. We can anticipate already at this stage that the field equations will contain terms involving the first derivative $\partial R / \partial b_{a}^{i}$ of the potential $R(h)$.

\subsection{Field equations}

The set of equations obtained by varying the full action of the theory (given by (2.7) plus the material action) with respect to $A^{i}$ (assuming that the material action does not depend on $A^{i}$ ) is

$$
d B^{i}+\epsilon^{i j k} A^{j}(B) \wedge B^{k}=0 .
$$

These equations are solved by

$$
A_{\mu}^{i}(B):=\frac{1}{2 \operatorname{det} B} B^{i \rho \sigma} B_{\rho \mu}^{j} \nabla^{\nu} B_{\nu \sigma}^{j}, \quad \operatorname{det} B:=-\frac{1}{24} \epsilon^{i j k} B_{\mu}^{i \nu} B_{\nu}^{j \rho} B_{\rho}^{k \mu} .
$$


We note that this connection is conformally-invariant and has the correct transformation properties of an $\mathrm{SO}(3)$ connection. That is, when the two-form field $B_{\mu \nu}^{i}$ transforms as $\delta B_{\mu \nu}^{i}=\epsilon^{i j k} \omega^{j} B_{\mu \nu}^{k}$, the connection transforms as $\delta_{\omega} A_{\mu}^{i}=\epsilon^{i j k} \omega^{j} A_{\mu}^{k}-\partial_{\mu} \omega^{i}$. A demonstration of this involves a simple identity satisfied by the two-forms $B_{\mu \nu}^{i}$; see [5]. We also note that, in most cases, instead of using (2.18) for finding $A^{i}(B)$, it is easier to solve (2.17) directly.

Once the connection $A^{i}(B)$ is found, one computes its curvature:

$$
F^{i}:=d A^{i}+\frac{1}{2} \epsilon^{i j k} A^{j} \wedge A^{k} .
$$

The field equations then read, in the parametrization (2.6):

$b_{a}^{i} F^{i}[A(b \Sigma)]=\left(\frac{\partial U}{\partial H^{i j}} b_{a}^{i} b_{b}^{j}+\frac{1}{3} \Lambda b_{a}^{i} b_{b}^{i}\right) \Sigma^{b}+2 \pi G T\left(\frac{\partial U_{m}}{\partial H^{i j}} b_{a}^{i} b_{b}^{j}+\frac{1}{3} \Lambda_{m} b_{a}^{i} b_{b}^{i}\right) \Sigma^{b}-2 \pi G T_{a b} \bar{\Sigma}^{b}$.

Here, $\Lambda$ and $\Lambda_{m}$ are the Legendre transforms

$$
\Lambda=U(H)-\frac{\partial U}{\partial H^{i j}} H^{i j}, \quad \Lambda_{m}=U_{m}(H)-\frac{\partial U_{m}}{\partial H^{i j}} H^{i j}
$$

of the potentials $U(H)$ and $U_{m}(H)$, respectively, $T$ is the trace of the stress-energy tensor of matter, $T_{a b}$ are the components of the traceless part of the stress-energy tensor, and $\bar{\Sigma}^{a}$ are the anti-self-dual metric two-forms (2.5). For the matter in the form of ideal fluid, we have

$$
T=(\rho-3 p), \quad T_{a b}=(\rho+p)\left(\frac{\delta_{a b}}{1-|u|^{2}}+2 \mathrm{i} \epsilon_{a b c} \frac{u^{c}}{\sqrt{1-|u|^{2}}}\right)-3 \mathcal{S}_{a b},
$$

where $\rho$ and $p$ are the energy density and pressure, respectively, $u^{a}$ is (proportional to) the momentum vector, and $\mathcal{S}_{a b}$ is the traceless matrix describing shear.

As anticipated in the previous subsection, the right-hand side of our field equations includes a term proportional to the trace $T$ of the stress-energy tensor and containing the first derivative of the potential function $R(h)$ ( or $U_{m}(H)$ ) of the material sector. The remainder of the dependence of the right-hand side of (2.20) on the stress-energy tensor components can be easily understood by working out how matter couples to gravity in the so-called Plebański formulation of GR; see, e.g., [8]. The only change in the present case as compared to GR in the Plebański formulation is in the appearance of the derivative terms $\partial U_{m}(H) / \partial H$. A detailed derivation of the field equations (2.20) can be found in [7].

In (2.21), we have introduced the "cosmological function" $\Lambda$ as the Legendre transform of $U(H)$. When $H^{i j}=0$, the above field equations reduce to those of GR in Plebański formulation. For later purposes, we note that the function $\Lambda$ is, in fact, a function $\Lambda(\Psi)$ of the quantity

$$
\Psi \equiv \Psi^{i j}:=\frac{\partial U}{\partial H^{i j}}
$$

that can be related to the usual Weyl curvature (at least for small deviations from GR). It is equally possible to parameterize the theory either by the original potential $U(H)$ or by its Legendre transform $\Lambda(\Psi)$. However, as we shall discuss in more detail a little below, 
the nature of the modification becomes more clear in the $\Lambda(\Psi)$ parametrization. This is due to the fact that the field $H^{i j}$ that measures the departure from GR arises in this parametrization as the first derivative

$$
H^{i j}=-\frac{\partial \Lambda}{\partial \Psi^{i j}}
$$

of the cosmological function $\Lambda$. Thus, for a function $\Lambda(\Psi)$ which is constant in its domain to a high degree, the deviations from GR are small.

\subsection{Large-distance modifications}

As we have already mentioned in the introduction, the class of theories under investigation can be viewed as ultraviolet modifications of gravity. This is most clearly seen in the parametrization of the theory by the potential $U(H)$. When it is expanded around $H^{i j}=0$, the first non-trivial term contains a mass parameter $m^{2}=1 / \ell^{2}$, and modifications away from GR only manifest themselves at energies $E$ larger than $m$. The low-energy limit $E \ll m$ of any of our theories is given by GR. However, when the theory is looked at non-perturbatively, e.g., in the Hamiltonian formulation (see [9]), one finds that the parametrization by the Legendre transform $\Lambda(\Psi)$ of $U(H)$ is more natural. More specifically, one finds that the departure of the theory from GR is connected with the fact that the cosmological constant becomes a function $\Lambda(\Psi)$ of what in GR used to be the Weyl part of the Riemann curvature tensor. The parametrization by $\Lambda(\Psi)$ then allows one to arrange for modification of gravity at any desirable scale of curvatures while keeping it close to GR at the scales of the Solar System. Indeed, the theory behaves close to GR whenever the first derivative (2.24) of the function $\Lambda(\Psi)$ is small. Thus, to be consistent with the Solar-System tests of gravity, one should assume the function $\Lambda(\Psi)$ to be approximately constant in the range of curvatures probed in the solar neighborhood. One can then allow $\Lambda(\Psi)$ to vary in the range of curvatures much smaller than those in the Solar System, which will produce deviations from GR at large distances (see [4] as well as a more accessible exposition [10]). Thus, according to our modification scenario, the value of the cosmological constant in the Solar System can be different from the value $\Lambda_{0}$ measured in cosmology, with the function $\Lambda(\Psi)$ interpolating between these two values. The parameter $\ell^{2}$ arising in (2.10) is then given by the second derivative of the function $\Lambda(\Psi)$ at $\Psi^{i j}=0$. Since gravity is not tested directly at very large distances, there are only very weak direct constraints on this parameter. As we shall see, a study of the physics of the cosmic microwave background (CMB) and formation of the large-scale structure as predicted by this theory will place much stronger constraints on the value of $\ell$.

Another important point that we would like to make is in the following. A detailed analysis shows that, as the first derivative (2.24) of $\Lambda(\Psi)$ becomes of order unity (in the sense that any of the eigenvalues of the symmetric traceless matrix $H^{i j}$ become of order one), the theory under consideration develops a specific degeneracy, which results in a singularity of metric (2.2) [4]. It can also be argued that it is no longer consistent to use the classical theory in this regime because quantum effects become important. Thus, it is necessary to avoid this regime if one wants to remain in the domain of applicability of the 
classical physics, which is usually the case for large distances of relevance for cosmology. Therefore, the classical theory will remain applicable only when the condition

$$
\left|\frac{\partial \Lambda}{\partial \Psi^{i j}}\right| \ll 1
$$

is satisfied. This condition can be rephrased by saying that the departure from GR as measured by the quantity (2.24) must be small. We assume this condition in what follows.

\subsection{The cosmological constant and vacuum energy}

In general relativity, the cosmological constant $\Lambda$ can be regarded as corresponding to the energy density of the vacuum, and one is free to absorb it in the energy density and pressure or into the scalar-field potential $V(\varphi)$ in all equations without exception. That is, the transformation

$$
\Lambda \rightarrow \Lambda-\lambda, \quad \rho \rightarrow \rho+\frac{\lambda}{8 \pi G}, \quad p \rightarrow p-\frac{\lambda}{8 \pi G}
$$

in the case of ideal fluid or

$$
\Lambda \rightarrow \Lambda-\lambda, \quad V(\varphi)+\frac{\lambda}{8 \pi G}
$$

in the case of scalar field leaves invariant all equations of the theory.

In the theory under consideration, this property is true only for the equations describing the homogeneous and isotropic universe, which are the same as in general relativity. It is not true in the general case, which can be seen in (2.20), in which the trace of the stress-energy tensor of matter $T$ enters differently from the genuine cosmological constant $\Lambda_{0}$ [present in the "cosmological function" $\Lambda(\Psi)$ ]. This feature of the theory of modified gravity persists in the equations for cosmological perturbations, as we shall see below. An exception is the special case when the parameter $\ell^{2}$ is fixed to be $\ell^{2}=1 / 4 g \Lambda_{0}$. In this case, the invariance with respect to (2.26) is restored at the level of linear perturbations.

\section{Homogeneous and isotropic universe}

Consider a homogeneous and isotropic background described by the two-forms $B_{0}^{i}$. On such a background, the traceless matrix $H^{i j}$ describing the departure from GR is equal to zero due to the spacetime symmetries. Thus, we have $H_{0}^{i j}=0$, and we can choose the scalars $b_{0 a}^{i}=\delta_{a}^{i}$, so that $B_{0}^{i}=\Sigma_{0}^{i}$. The background equations are then the Friedmann equations in the Plebański formalism:

$$
F^{i}\left[A\left(\Sigma_{0}\right)\right]=\frac{2 \pi G T_{0}+\Lambda_{0}}{3} \Sigma_{0}^{i}-2 \pi G \bar{T}_{0} \bar{\Sigma}_{0}^{j}
$$

Here,

$$
T_{0}:=(\rho-3 p), \quad \bar{T}_{0}:=(\rho+p)
$$

are the components of the background stress-energy tensor. We emphasize that the background evolves in our theory in exactly the same way as in GR. Modifications only arise 
in the dynamics of perturbations. This is, however, the story for an ideally homogeneous backgrounds. In reality, there are inhomogeneities on various scales that need to be averaged over. It is natural to expect that this will introduce modifications of the background equations as well, to be taken into account. However, since the averaging issue is not fully understood even in the simpler case of GR, we postpone such an analysis to our future publications, and, in this paper, study the cosmological perturbations around an ideally homogeneous and isotropic background.

In the conformal time $\eta$, the metric of a spatially flat homogenous and isotropic universe is:

$$
d s_{0}^{2}=a^{2}(\eta)\left[d \eta^{2}-\sum_{i}\left(d x^{i}\right)^{2}\right] .
$$

In what follows, we often omit the argument of the scale factor $a(\eta)$.

A set of self-dual and anti-self-dual two-forms describing this metric can be chosen as

$$
\Sigma_{0}^{i}=a^{2}\left(\mathrm{i} d \eta \wedge d x^{i}-\frac{1}{2} \epsilon^{i j k} d x^{j} \wedge d x^{k}\right), \quad \bar{\Sigma}_{0}^{i}=a^{2}\left(\mathrm{i} d \eta \wedge d x^{i}+\frac{1}{2} \epsilon^{i j k} d x^{j} \wedge d x^{k}\right) .
$$

The connection $A_{0}^{i}$ compatible with the set of self-dual two-forms $\Sigma_{0}^{i}$, i.e., satisfying $d \Sigma_{0}^{i}+$ $\epsilon^{i j k} A_{0}^{j} \wedge \Sigma_{0}^{k}=0$, is given by

$$
A_{0}^{i}=\mathrm{i} \mathcal{H} d x^{i}
$$

where

$$
\mathcal{H}:=\frac{a^{\prime}}{a},
$$

and the prime denotes the derivative with respect to the conformal time $\eta$. The curvature $F^{i}=d A^{i}+\frac{1}{2} \epsilon^{i j k} A^{j} \wedge A^{k}$ of connection (3.5) is given by

$$
F_{0}^{i}=\frac{1}{2 a^{2}}\left(\mathcal{H}^{\prime}+\mathcal{H}^{2}\right) \Sigma^{i}+\frac{1}{2 a^{2}}\left(\mathcal{H}^{\prime}-\mathcal{H}^{2}\right) \bar{\Sigma}^{i}
$$

where we have used the relations

$$
d \eta \wedge d x^{i}=\frac{1}{2 \mathrm{i} a^{2}}\left(\Sigma_{0}^{i}+\bar{\Sigma}_{0}^{i}\right), \quad d x^{i} \wedge d x^{j}=\frac{1}{2 a^{2}} \epsilon^{i j k}\left(\bar{\Sigma}_{0}^{k}-\Sigma_{0}^{k}\right)
$$

to decompose $F_{0}^{i}$ into its self-dual and anti-self-dual parts.

Substituting (3.7) into (3.1), we obtain the dynamical equations for the background. The condition that the self-dual and anti-self-dual parts of the field equations are separately satisfied gives two equations whose symbolic linear combinations $\Sigma-\bar{\Sigma}$ and $3 \Sigma+\bar{\Sigma}$ are just the Friedmann equations

$$
\frac{\mathcal{H}^{2}}{a^{2}}=\frac{8 \pi G \rho}{3}+\frac{\Lambda_{0}}{3}, \quad \frac{2 \mathcal{H}^{\prime}+\mathcal{H}^{2}}{a^{2}}=-8 \pi G p+\Lambda_{0} .
$$

\section{Linearized field equations}

Linearizing the field equations (2.20) around the homogeneous and isotropic background, we denote the perturbation of a quantity by a symbol $\delta$ next to the symbol representing 
it. There arises a perturbation of the metric two-forms $\delta \Sigma^{i}$ as well as a perturbation of the scalars $\delta b_{a}^{i}$. Using the fact that $B_{0 a}^{i}=\delta_{a}^{i}$, now we can identify the two types of $\operatorname{SO}(3)$ indices. Thus, from now on we drop the distinction between the two types, and use only the $i, j, k$ indices. The condition $R\left(h^{i j}\right)=1$ implies, at the first order in perturbation, that the matrix $\delta b^{i j}$ is traceless. The perturbations of the metric $h^{i j}$ and of its trace-free part are then $\delta h^{i j}=\delta H^{i j}=2 \delta b^{(i j)}$. Since $H_{0}^{i j}=0$, the first-order perturbation of the quantity $\operatorname{Tr} H^{2}$ that appears in the potential functions is zero. Thus, $\delta \Lambda=0$ and $\delta \Lambda_{m}=0$.

After some algebra, the linearized field equations are found to be

$$
\delta F^{i}+\delta b^{i j} F_{0}^{j}=\frac{2 \pi G}{3} \delta T \Sigma_{0}^{i}-2 \pi G \delta T^{i j} \bar{\Sigma}_{0}^{j}+\frac{2 \pi G T_{0}+\Lambda_{0}}{3} \delta \Sigma^{i}-2 \pi G \bar{T}_{0} \delta \bar{\Sigma}^{i}-4 \pi G \kappa \delta b^{i j} \Sigma_{0}^{j} .
$$

Only the last terms on the left-hand side and on the right-hand side of this equation are not present in GR, with all other terms being exactly like in the Plebański formulation of GR. The perturbation of the components of the stress-energy tensor of fluid is given by

$$
\delta T=\delta \rho-3 \delta p, \quad \delta T^{i j}=(\delta \rho+\delta p) \delta^{i j}+2 \mathrm{i} \epsilon^{i j k} \delta u^{k}-3 \delta \mathcal{S}^{i j},
$$

where, for convenience, we have absorbed the factor $\rho+p$ into the momentum vector $u^{i}$. The quantity $\delta F^{i}$ is the linearized curvature corresponding to the perturbation of the $B^{i}$ field given by

$$
\delta B^{i}=\delta b^{i j} \Sigma_{0}^{j}+\delta \Sigma^{i}
$$

where $\delta \Sigma^{i}$ is the perturbation of the metric self-dual two-forms (see below for explicit expressions). In (4.1), we have introduced a new parameter $\kappa$ with dimensions of the energy density:

$$
\kappa:=\left(g-\frac{1}{3}\right) T_{0}+\frac{1}{8 \pi G}\left(\frac{1}{\ell^{2}}-\frac{4}{3} \Lambda_{0}\right)=\beta(\rho-3 p)+\gamma \rho_{\Lambda},
$$

where $T_{0}=\rho-3 p$ is the trace of the material stress-energy tensor, $\rho_{\Lambda}=\Lambda_{0} / 8 \pi G$ is the energy density of the cosmological constant, and $\ell$ and $g$ are defined in (2.10) and (2.12), respectively. The quantities $\beta$ and $\gamma$ are two new convenient dimensionless parameters:

$$
\beta=g-\frac{1}{3}, \quad \gamma=\frac{1}{\ell^{2} \Lambda_{0}}-\frac{4}{3} .
$$

These parameters have the following meaning. The parameter $\gamma$ determines the deviation of the gravitational potential $V\left(h^{i j}\right)$ from one given by $V\left(h^{i j}\right)=\Lambda_{0} R\left(h^{i j}\right)$. This case corresponds to $4 \beta=\gamma$ and describes the theory that couples in the same way to the cosmological constant and to the trace of the stress-energy tensor. The parameter $\beta$ controls the deviation of the physical metric from the so-called Urbantke metric which coincides with (2.2) precisely. The value $\beta=0$ corresponds to coupling of matter to the Urbantke metric. Finally, the limit to GR is obtained by keeping $\beta$ fixed (and arbitrary), and sending $\ell \rightarrow 0$ (thus $\gamma \rightarrow \infty$ ) keeping the product $\gamma \delta B^{i j}$ fixed. To summarize, schematically we have:

$$
\beta=0 \Longleftrightarrow \text { coupling to the Urbantke metric, } \quad \gamma \rightarrow \infty \Longleftrightarrow \text { limit to GR . }
$$


Now, using the expression (3.7) for the background curvature and equations (3.1), we get

$$
\frac{2 \pi G T_{0}+\Lambda_{0}}{3}=\frac{\mathcal{H}^{2}+\mathcal{H}^{\prime}}{2 a^{2}}, \quad 2 \pi G \bar{T}_{0}=\frac{\mathcal{H}^{2}-\mathcal{H}^{\prime}}{2 a^{2}} .
$$

The linear equations for perturbations can then be written in the form

$$
\begin{aligned}
& \delta B^{i j} F_{0}^{j}+\delta F^{i}-\frac{1}{2 a^{2}}\left(\mathcal{H}^{2}+\mathcal{H}^{\prime}\right) \delta \Sigma^{i}+\frac{1}{2 a^{2}}\left(\mathcal{H}^{2}-\mathcal{H}^{\prime}\right) \delta \bar{\Sigma}^{i} \\
& =\frac{2 \pi G}{3} \delta T \Sigma_{0}^{i}-2 \pi G \delta T^{i j} \bar{\Sigma}_{0}^{j}-4 \pi G \kappa \delta B^{i j} \Sigma_{0}^{j},
\end{aligned}
$$

where the stress-energy perturbations of the fluid are given, as before, by

$$
\delta T=(\delta \rho-3 \delta p), \quad \delta T^{i j}=(\delta \rho+\delta p) \delta^{i j}+2 \mathrm{i} \epsilon^{i j k} \delta u^{k}-3 \delta \mathcal{S}^{i j},
$$

and $\delta B^{i j}$ is the non-metric part of the perturbation (see the next section for explicit expressions). This form of the linearized equations is most convenient for further calculations.

\section{Classification of perturbations}

A general infinitesimal perturbation of the background (3.4) can be decomposed into the background two-forms:

$$
\delta B^{i}=\delta \Phi^{i j} \Sigma_{0}^{j}+\delta \Upsilon^{i j} \bar{\Sigma}_{0}^{j} .
$$

However, it is clear that much of this perturbation is a gauge transformation. Indeed, the anti-symmetric part $\delta \Phi^{[i j]}$ describes an infinitesimal $\mathrm{SO}(3)$ rotation of the triple of two-forms $\Sigma^{i}$ and is thus a pure gauge. Moreover, some part of the general perturbation (5.1) is an infinitesimal diffeomorphism of the background (3.4). To separate the physical quantities from the gauge ones, we thus need to compute the effect of an infinitesimal diffeomorphism on the background (3.4). The Lie derivative of $B^{i}$, suitably corrected by a gauge transformation, can be shown to be given by $\mathcal{L}_{\xi} B^{i}=\mathcal{D} B^{i}(\xi)$, where $B^{i}(\xi)$ is the interior multiplication of a vector field $\xi$ with two-form $B^{i}$, and $\mathcal{D}$ is the covariant derivative with respect to the $B$-compatible connection. For the background (3.4), a straightforward but lengthy calculation gives

$$
\begin{aligned}
\mathcal{L}_{\xi} \Sigma_{0}^{i}= & \frac{1}{2}\left[4 \mathcal{H} \xi^{0}+\xi^{0 \prime}+\Delta \zeta\right] \Sigma_{0}^{i}+\xi_{[i, j]} \Sigma_{0}^{j}+\frac{\mathrm{i}}{2} \epsilon^{i j k}\left[2 \mathcal{H}\left(\xi_{j}+\zeta_{, j}\right)+\left(\xi_{j}+\zeta_{, j}\right)^{\prime}+\xi^{0}, j\right] \Sigma_{0}^{k} \\
& +\frac{1}{2}\left[\xi^{0 \prime}-\Delta \zeta\right] \bar{\Sigma}_{0}^{i}+\left[\xi_{(i, j)}+\zeta_{, i j}\right] \bar{\Sigma}_{0}^{j}+\frac{\mathrm{i}}{2} \epsilon^{i j k}\left[\left(\xi_{j}+\zeta_{, j}\right)^{\prime}-\xi_{, j}^{0}\right] \bar{\Sigma}_{0}^{k} .
\end{aligned}
$$

Here, we have decomposed the vector field $\xi$ into its components $\xi=\left(\xi^{0}, \xi_{i}+\zeta_{, i}\right)$, where $\xi_{i}$ is transverse $\xi_{, i}^{i}=0$. We see that the effect of a diffeomorphism is a change in the metric described by $\Sigma_{0}^{i}$ together with a gauge transformation on $\Sigma_{0}^{i}$. The latter can always be corrected by an $\mathrm{SO}(3)$ rotation, which allows us to drop the anti-symmetric part of the tensor $\delta \Phi^{i j}$ from now on. Note that diffeomorphisms do not affect the symmetric traceless part of $\delta \Phi^{i j}$, i.e., they do not act on the non-metric ${ }^{3}$ components of the $B^{i}$ field.

\footnotetext{
${ }^{3}$ We shall often refer to the non-dynamical scalars present in $B^{i}$ in addition to the metric as its "nonmetric" components.
} 
In principle, this could have been expected, for they act in the spacetime manifold, not in the internal space, where the non-metric components of $B^{i}$ reside.

Let us now consider the usual classification of perturbations into scalar, vector and tensor sectors and deduce the most general form of these perturbations after all gauge freedom is fixed.

\subsection{Scalar sector}

Scalar perturbations are described by scalar functions, so that we have

$$
\begin{aligned}
& \delta \Phi^{i j}=\phi \delta^{i j}+\frac{1}{a^{2}}\left(\chi_{, i j}-\frac{1}{3} \delta_{i j} \Delta \chi\right)+\frac{\mathrm{i}}{4} \epsilon^{i j k} \theta_{, k}, \\
& \delta \Upsilon^{i j}=\psi \delta^{i j}+\frac{1}{a^{2}}\left(\sigma_{, i j}-\frac{1}{3} \delta_{i j} \Delta \sigma\right)+\frac{\mathrm{i}}{4} \epsilon^{i j k} \omega_{, k} .
\end{aligned}
$$

Here, we have introduced the factors $1 / a^{2}$ for future convenience, $\phi$ and $\psi$ are the trace parts, $\chi$ and $\sigma$ are the scalar parts of the traceless symmetric parts, and $\theta$ and $\omega$ are the scalar parts of the anti-symmetric parts of the self-dual and anti-self-dual perturbations, respectively. It is easy to see that diffeomorphism (5.2) can be used to set the scalars $\sigma$ and $\omega$ to zero, while an $\mathrm{SO}(3)$ gauge transformation can be used to set to zero the scalar $\theta$. Thus, we are led to consider the following "physical" scalar perturbations:

$$
\delta B^{i}=\delta B^{i j} \Sigma_{0}^{j}+\phi \Sigma_{0}^{i}+\psi \bar{\Sigma}_{0}^{i}
$$

where we have introduced a convenient notation for the non-metric part

$$
\delta B^{i j}=\frac{1}{a^{2}}\left(\chi_{, i j}-\frac{1}{3} \delta_{i j} \Delta \chi\right)
$$

In other words, the scalar perturbation (5.5) is a sum of a non-metric perturbation $\delta B^{i j} \Sigma_{0}^{j}$ and the usual metric perturbation

$$
\delta \Sigma^{i}=\phi \Sigma_{0}^{i}+\psi \bar{\Sigma}_{0}^{i}
$$

The potential $\chi$ can, in general, be complex, while $\phi$ and $\psi$ are real. The potential $\chi$ does not have a counterpart in GR, while the two potentials $\phi$ and $\psi$ have a very simple relation to the standard GR potentials appearing in the scalar perturbation of the metric:

$$
d s^{2}=a^{2}\left[\left(1+2 \Phi_{\mathrm{GR}}\right) d \eta^{2}-\left(1-2 \Psi_{\mathrm{GR}}\right) \sum_{i}\left(d x^{i}\right)^{2}\right] .
$$

An elementary calculation shows the relation between $\Phi_{\mathrm{GR}}, \Psi_{\mathrm{GR}}$ and the potentials $\phi, \psi$ in (5.5):

$$
\Phi_{\mathrm{GR}}=\frac{3 \psi+\phi}{2}, \quad \Psi_{\mathrm{GR}}=\frac{\psi-\phi}{2} .
$$




\subsection{Vector sector}

The most general perturbation of the vector type can be reduced to

$$
\delta B^{i}=2\left[\zeta^{(i, j)} \Sigma_{0}^{j}+\xi^{(i, j)} \bar{\Sigma}_{0}^{j}\right]
$$

where the vector potentials $\zeta^{i}$ and $\xi^{i}$ are transverse, $\zeta^{i}{ }_{, i}=\xi^{i}{ }_{, i}=0$, and $\zeta^{i}$ may be complex. Alternatively, it is a sum of a non-metric perturbation $\delta B^{i j} \Sigma_{0}^{j}$ with

$$
\delta B^{i j}=2 \zeta^{(i, j)}
$$

and a metric one:

$$
\delta \Sigma^{i}=2 \xi^{(i, j)} \bar{\Sigma}_{0}^{j}
$$

\subsection{Tensor sector}

Perturbations of the tensor type that correspond to gravitons are given by

$$
\delta B^{i}=\frac{1}{a^{2}} \chi^{i j} \Sigma^{j}+\rho^{i j} \bar{\Sigma}^{j}
$$

where both $\chi^{i j}$ and $\rho^{i j}$ are symmetric, traceless and transverse $\left(\chi^{i j}{ }_{i}=\rho^{i j}{ }_{, i}=0\right)$ matrices, and $\chi^{i j}$ may be complex while $\rho^{i j}$ is real (see, however, Appendix). Again, the perturbation is a sum of a non-metric one

$$
\delta B^{i j}=\frac{1}{a^{2}} \chi^{i j}
$$

and a metric one

$$
\delta \Sigma^{i}=\rho^{i j} \bar{\Sigma}_{0}^{j}
$$

\section{Scalar perturbations}

\subsection{Curvature}

In this section, we consider the important case of scalar perturbations in detail. First of all, we compute the connections and then (linearized) curvatures for the perturbations described by $\phi, \psi$, and $\chi$. To compute the perturbation of the $B$-compatible connection for a perturbation $\delta B^{i}$, one has to solve the algebraic equations $\mathcal{D}_{0}\left(\delta B^{i}\right)+\epsilon^{i j k} \delta A^{j} \wedge \Sigma_{0}^{k}=0$, where $\Sigma_{0}^{i}$ is the background two-form. The curvature of an infinitesimal connection $\delta A^{i}$ is then given by $F^{i}(\delta A)=\mathcal{D}_{0} \delta A^{i}$, where $\mathcal{D}_{0}$ is the covariant derivative with respect to the background connection. The computations are straightforward, but rather lengthy. The expressions for the connection components are:

$$
\begin{aligned}
\delta A_{\phi}^{i} & =\frac{\mathrm{i}}{2} \phi_{, i} d \eta+\frac{1}{2}\left(\mathrm{i} \phi^{\prime} \delta_{i j}-\epsilon_{i j k} \phi_{, k}\right) d x^{j} \\
\delta A_{\psi}^{i} & =\frac{3 \mathrm{i}}{2} \psi_{, i} d \eta-\frac{1}{2}\left[\mathrm{i} \frac{\left(a^{4} \phi\right)^{\prime}}{a^{4}} \delta_{i j}-\epsilon_{i j k} \phi_{, k}\right] d x^{j}, \\
\delta A_{\chi}^{i} & =-\frac{2 \mathrm{i}}{3 a^{2}} \Delta \chi_{, i} d \eta-\frac{1}{3} \epsilon_{i j k} \Delta \chi_{, k} d x^{j}-\frac{\mathrm{i}}{a^{3}}\left(a \chi_{, i j}-\frac{a}{3} \Delta \chi \delta_{i j}\right)^{\prime} d x^{j} .
\end{aligned}
$$


The expressions for the curvatures, decomposed into their self-dual and anti-self-dual parts, are

$$
\begin{aligned}
\mathcal{D} \delta A_{\phi}^{i}= & \frac{1}{4 a^{2}}\left(\phi^{\prime \prime}-\Delta \phi+2 \mathcal{H} \phi^{\prime}\right) \Sigma_{0}^{i} \\
& +\frac{1}{4 a^{2}}\left[-2 \phi_{, i j}+\delta_{i j}\left(\phi^{\prime \prime}+\Delta \phi\right)+2 i \epsilon_{i j k}\left(\frac{\phi_{, k}}{a}\right)^{\prime} a-2 \mathcal{H} \phi^{\prime} \delta_{i j}\right] \bar{\Sigma}_{0}^{j} \\
\mathcal{D} \delta A_{\psi}^{i}= & -\frac{1}{4 a^{2}}\left[4 \psi_{, i j}+\delta_{i j}\left(\psi^{\prime \prime}-\Delta \psi\right)+4 \delta_{i j}\left(\mathcal{H}^{\prime} \psi+2 \mathcal{H}^{2} \psi+\frac{3}{2} \mathcal{H} \psi^{\prime}\right)\right] \Sigma_{0}^{j} \\
& -\frac{1}{4 a^{2}}\left[2 \psi_{, i j}+\delta_{i j}\left(\psi^{\prime \prime}+\Delta \psi\right)+4 \delta_{i j}\left(\mathcal{H}^{\prime} \psi-2 \mathcal{H}^{2} \psi+\frac{1}{2} \mathcal{H} \psi^{\prime}\right)\right. \\
& \left.+2 \mathrm{i} \epsilon_{i j k} \frac{\left(a^{3} \psi_{, k}\right)^{\prime}}{a^{3}}\right] \bar{\Sigma}_{0}^{j}, \\
\mathcal{D} \delta A_{\chi}^{i}= & -\frac{1}{2 a^{4}}\left[\left(\partial_{\eta}^{2}-2 \mathcal{H} \partial_{\eta}-\Delta-4 \mathcal{H}^{2}\right)\left(\chi, i j-\frac{1}{3} \delta_{i j} \Delta \chi\right)\right] \Sigma_{0}^{j} \\
& -\frac{1}{2 a^{4}}\left[\left(\partial_{\eta}^{2}-\frac{1}{3} \Delta\right)\left(\chi_{, i j}-\frac{1}{3} \delta_{i j} \Delta \chi\right)\right] \bar{\Sigma}_{0}^{i} \\
& +\frac{\mathrm{i}}{3 a^{4}} \epsilon_{i j k} \Delta \chi_{, k}^{\prime} \bar{\Sigma}_{0}^{j}+\frac{2}{9 a^{4}} \Delta^{2} \chi \bar{\Sigma}_{0}^{i}-\delta B^{i j} F_{0}^{j}
\end{aligned}
$$

where $\delta B^{i j}$ in the last formula is given by (5.6), and $F_{0}^{i}$ is the background curvature (3.7). The differential operators in brackets act only on $\chi$ but not on the background forms $\Sigma_{0}^{i}$.

\subsection{Field equations}

Now we can substitute the expressions for the curvatures obtained above into the linearized field equations (4.8). For the scalar sector, the sources are

$$
\delta T=\delta \rho-3 \delta p, \quad \delta T^{i j}=(\delta \rho+\delta p) \delta^{i j}+\frac{2 \mathrm{i}}{a} \epsilon^{i j k} \delta u_{, k}-3\left(\delta \mathcal{S}_{, i j}-\frac{1}{3} \delta_{i j} \Delta \delta \mathcal{S}\right),
$$

where we have introduced a convenient normalization of the scalar part of the velocity perturbation $\delta u$. The resulting equations are most usefully separated into the symmetric traceless, antisymmetric, and trace parts of the matrices arising in front of the background two-forms $\Sigma_{0}^{i}$ and $\bar{\Sigma}_{0}^{i}$. Let us first analyze the symmetric trace-free parts. After removing the directional derivatives and multiplying by $a^{2}$ in the self-dual sector, and by $2 a^{2}$ in the anti-self-dual sector, the self-dual and anti-self-dual parts give, respectively,

$$
\begin{aligned}
& \psi+\frac{1}{2 a^{2}}\left(\chi^{\prime \prime}-2 \mathcal{H} \chi^{\prime}-\Delta \chi-4 \mathcal{H}^{2} \chi\right)=4 \pi G \kappa \chi, \\
& \phi+\psi+\frac{1}{a^{2}}\left(\chi^{\prime \prime}-\frac{1}{3} \Delta \chi\right)=-12 \pi G a^{2} \delta \mathcal{S} .
\end{aligned}
$$

It is more convenient, however, to consider these equations as those determining the usual relativistic potentials $\Psi_{\mathrm{GR}}$ and $\Phi_{\mathrm{GR}}$; see (5.9). We have

$$
\begin{aligned}
\Psi_{\mathrm{GR}} & :=\frac{\psi-\phi}{2}=4 \pi G \kappa \chi+\frac{1}{a^{2}}\left(\frac{1}{3} \Delta \chi+\mathcal{H} \chi^{\prime}+2 \mathcal{H}^{2} \chi\right)+6 \pi G a^{2} \delta \mathcal{S}, \\
\Phi_{\mathrm{GR}} & :=\frac{\phi+3 \psi}{2}=4 \pi G \kappa \chi+\frac{1}{a^{2}}\left(-\chi^{\prime \prime}+\frac{2}{3} \Delta \chi+\mathcal{H} \chi^{\prime}+2 \mathcal{H}^{2} \chi\right)-6 \pi G a^{2} \delta \mathcal{S} .
\end{aligned}
$$


The difference between the relativistic potentials is given by

$$
\Psi_{\mathrm{GR}}-\Phi_{\mathrm{GR}}=\frac{1}{a^{2}}\left(\chi^{\prime \prime}-\frac{1}{3} \Delta \chi\right)+12 \pi G a^{2} \delta \mathcal{S} .
$$

Thus, even in the absence of shear, the relativistic potentials $\Psi_{\mathrm{GR}}$ and $\Phi_{\mathrm{GR}}$ are not equal to each other in our theory, which is typical of modified gravity.

The antisymmetric part is only present in the anti-self-dual sector. After removing the directional derivative and multiplying by $\mathrm{i} a^{2}$, one obtains

$$
\Psi_{\mathrm{GR}}^{\prime}+\mathcal{H} \Phi_{\mathrm{GR}}-\frac{1}{3 a^{2}} \Delta \chi^{\prime}=4 \pi G a \delta u
$$

which, after solving for the perturbations $\Psi_{\mathrm{GR}}, \Phi_{\mathrm{GR}}$, and $\chi$, will determine the scalar part of the 4 -velocity perturbation. Finally, the trace parts of the arising equations are most convenient in their schematic linear combinations $\Sigma-\bar{\Sigma}$ and $3 \Sigma+\bar{\Sigma}$. Using the equations of motion for the background, after some simple algebra, we get

$$
\begin{aligned}
& \Delta \Psi_{\mathrm{GR}}-3 \mathcal{H} \Psi_{\mathrm{GR}}^{\prime}-3 \mathcal{H}^{2} \Phi_{\mathrm{GR}}-\frac{1}{3 a^{2}} \Delta^{2} \chi=4 \pi G a^{2} \delta \rho, \\
& \Psi_{\mathrm{GR}}^{\prime \prime}+3 \mathcal{H} \Psi_{\mathrm{GR}}^{\prime}+\left(2 \mathcal{H}^{\prime}+\mathcal{H}^{2}\right) \Psi_{\mathrm{GR}}+\frac{1}{3} \Delta\left(\Phi_{\mathrm{GR}}-\Psi_{\mathrm{GR}}\right)+\mathcal{H}\left(\Phi_{\mathrm{GR}}-\Psi_{\mathrm{GR}}\right)^{\prime} \\
& +\left(2 \mathcal{H}^{\prime}+\mathcal{H}^{2}\right)\left(\Phi_{\mathrm{GR}}-\Psi_{\mathrm{GR}}\right)-\frac{1}{9 a^{2}} \Delta^{2} \chi=4 \pi G a^{2} \delta p .
\end{aligned}
$$

Thus, the arising equations are exactly like in GR apart from the presence of simple additional terms containing $\chi$.

\subsection{Conservation equations}

The following standard conservation equations hold in the theory under consideration:

$$
\begin{aligned}
& \delta \rho^{\prime}+3 \mathcal{H}(\delta \rho+\delta p)=3(\rho+p) \Psi_{\mathrm{GR}}^{\prime}+\frac{1}{a} \Delta \delta u \\
& \delta u^{\prime}+3 \mathcal{H} \delta u=a \delta p+a(\rho+p) \Phi_{\mathrm{GR}}+a \Delta \delta \mathcal{S} .
\end{aligned}
$$

This can be verified directly using equations (6.9), (6.10), and (6.13) above. Note that the field $\chi$, responsible for the modification, does not appear in these equations. This is of no surprise as the stress-energy is conserved in this theory in the usual way.

\subsection{Equations for the "non-metricity" scalar $\chi$}

At this point, the usual GR procedure would be to use the fact that $\Phi_{\mathrm{GR}}=\Psi_{\mathrm{GR}}$ (in the absence of shear) and obtain a system of equations for one of the potentials. In our case, this is not possible because any such equation will involve contributions containing the nonmetric scalar $\chi$. However, it is possible to obtain a closed system of equations for this scalar. Thus, we solve for $\Phi_{\mathrm{GR}}$ and $\Psi_{\mathrm{GR}}$ in terms of the new potential $\chi$ and deal with differential equations for $\chi$. The resulting equations are not simple, but it is worth presenting them here to show that their structure is similar to the corresponding equations 
in GR. Thus, we now substitute $\psi+\phi=\Phi_{\mathrm{GR}}-\Psi_{\mathrm{GR}}, \Psi_{\mathrm{GR}}$, and $\Phi_{\mathrm{GR}}$ from (6.9), (6.10), and (6.11), respectively, into (6.14) and (6.15), and, after some lengthy algebra, obtain

$$
\begin{aligned}
4 \pi G a^{2} \delta \rho= & \Delta(4 \pi G \kappa \chi)-3 \mathcal{H}(4 \pi G \kappa \chi)^{\prime}-3 \mathcal{H}^{2}(4 \pi G \kappa \chi) \\
& +\frac{2}{a^{2}}\left[\mathcal{H}^{2} \Delta \chi-\frac{3 \mathcal{H}}{2} \chi^{\prime}\left(\mathcal{H}^{\prime}+\mathcal{H}^{2}\right)+3 \mathcal{H}^{2}\left(\mathcal{H}^{2}-2 \mathcal{H}^{\prime}\right) \chi\right] \\
& +6 \pi G a^{2}\left[\Delta \delta \mathcal{S}-3 \mathcal{H}\left(\delta \mathcal{S}^{\prime}+\mathcal{H} \delta \mathcal{S}\right)\right] \\
4 \pi G a^{2} \delta p= & (4 \pi G \kappa \chi)^{\prime \prime}+3 \mathcal{H}(4 \pi G \kappa \chi)^{\prime}+\left(2 \mathcal{H}^{\prime}+\mathcal{H}^{2}\right)(4 \pi G \kappa \chi) \\
& +\frac{2}{a^{2}}\left[\mathcal{H}^{2} \chi^{\prime \prime}+\frac{1}{3} \Delta \chi\left(\mathcal{H}^{\prime}-\mathcal{H}^{2}\right)+\frac{1}{2} \chi^{\prime}\left(\mathcal{H}^{\prime \prime}+7 \mathcal{H}^{\prime} \mathcal{H}-3 \mathcal{H}^{3}\right)\right. \\
& \left.+\chi\left(2 \mathcal{H}^{\prime \prime} \mathcal{H}+2 \mathcal{H}^{\prime 2}-2 \mathcal{H}^{2} \mathcal{H}^{\prime}-\mathcal{H}^{4}\right)\right] \\
& +6 \pi G a^{2}\left[\delta \mathcal{S}^{\prime \prime}+5 \mathcal{H}\left(\delta \mathcal{S}^{\prime}+\mathcal{H} \delta \mathcal{S}\right)-\frac{2}{3} \Delta \delta \mathcal{S}\right] .
\end{aligned}
$$

Similarly, substituting $\Psi_{\mathrm{GR}}$ and $\Phi_{\mathrm{GR}}$ into (6.13), we get

$$
\begin{array}{r}
4 \pi G a \delta u=(4 \pi G \kappa \chi)^{\prime}+\mathcal{H}(4 \pi G \kappa \chi)+\frac{1}{a^{2}}\left[\chi^{\prime}\left(\mathcal{H}^{\prime}+\mathcal{H}^{2}\right)+2 \chi \mathcal{H}\left(2 \mathcal{H}^{\prime}-\mathcal{H}^{2}\right)\right] \\
+6 \pi G a^{2}\left(\delta \mathcal{S}^{\prime}+\mathcal{H} \delta \mathcal{S}\right)
\end{array}
$$

This equation determines the scalar part of the velocity perturbation once the perturbations $\chi$ and $\delta \mathcal{S}$ have been found.

Now, introducing the speed of sound $c_{s}$ via

$$
\delta p=c_{s}^{2} \delta \rho+\tau \delta \mathcal{E}
$$

we can write a single dynamical equation for $\chi$ with the entropy perturbation $\delta \mathcal{E}$ as the source. It reads:

$$
\begin{aligned}
4 \pi G a^{2} \tau \delta \mathcal{E}= & (4 \pi G \kappa \chi)^{\prime \prime}-c_{s}^{2} \Delta(4 \pi G \kappa \chi)+3 \mathcal{H}\left(1+c_{s}^{2}\right)(4 \pi G \kappa \chi)^{\prime} \\
+ & \left.+2 \mathcal{H}^{\prime}+\mathcal{H}^{2}\left(1+3 c_{s}^{2}\right)\right](4 \pi G \kappa \chi) \\
& +\frac{2}{a^{2}}\left(\mathcal{H}^{2} \chi^{\prime \prime}+\frac{1}{3} \Delta \chi\left[\mathcal{H}^{\prime}-\mathcal{H}^{2}\left(1+3 c_{s}^{2}\right)\right]\right. \\
& +\frac{\chi^{\prime}}{2}\left[\mathcal{H}^{\prime \prime}+\mathcal{H}^{\prime} \mathcal{H}\left(7+3 c_{s}^{2}\right)+3 \mathcal{H}^{3}\left(c_{s}^{2}-1\right)\right] \\
& \left.+\chi\left[2 \mathcal{H}^{\prime \prime} \mathcal{H}+2 \mathcal{H}^{\prime 2}-2 \mathcal{H}^{2} \mathcal{H}^{\prime}\left(1-3 c_{s}^{2}\right)-\mathcal{H}^{4}\left(1+3 c_{s}^{2}\right)\right]\right) \\
& +6 \pi G a^{2}\left[\delta \mathcal{S}^{\prime \prime}+\left(5+3 c_{s}^{2}\right) \mathcal{H}\left(\delta \mathcal{S}^{\prime}+\mathcal{H} \delta \mathcal{S}\right)-\left(\frac{2}{3}+c_{s}^{2}\right) \Delta \delta \mathcal{S}\right]
\end{aligned}
$$

In the absence of shear $(\delta \mathcal{S}=0)$, this dynamical equation can be solved for $\chi$, after which one can compute all other quantities of interest, such as the relativistic potentials and perturbations of energy density and pressure. 


\subsection{New relativistic potential}

The obtained complicated equations for $\chi$ can be considerably simplified by introducing a new quantity

$$
\Phi:=\left(4 \pi G \kappa+\frac{2 \mathcal{H}^{2}}{a^{2}}\right) \chi .
$$

One rationale for considering precisely this combination is the identity that follows directly from $(6.18)$ and $(6.20)$ :

$$
4 \pi G a^{2}\left(\delta \rho+\frac{3 \mathcal{H}}{a} \delta u-\frac{3}{2} \Delta \delta \mathcal{S}\right)=\Delta \Phi
$$

Precisely the same identity holds in the case of GR with $\Phi$ on the right-hand side of this equation being the relativistic potential $\Phi_{\mathrm{GR}}$, which suggests that the quantity $\Phi$ should play the role of the main relativistic potential in our theory. Below we shall see that this expectation is realized.

Anticipating the role that $\Phi$ is going to play, it is illuminating to rewrite the main dynamical equation (6.22) in terms of $\Phi$. Most of the terms in this equation combine into the usual GR-type equation for the potential $\Phi$. Few terms remain, however, and these are the contributions due to non-metricity. It is most convenient to write them in terms of $\chi$. We get:

$$
\begin{aligned}
4 \pi G a^{2} \tau \delta \mathcal{E}= & \Phi^{\prime \prime}-c_{s}^{2} \Delta \Phi+3 \mathcal{H}\left(1+c_{s}^{2}\right) \Phi^{\prime}+\left[2 \mathcal{H}^{\prime}+\mathcal{H}^{2}\left(1+3 c_{s}^{2}\right)\right] \Phi \\
& +6 \pi G a^{2}\left[\delta \mathcal{S}^{\prime \prime}+5 \mathcal{H}\left(\delta \mathcal{S}^{\prime}+\mathcal{H} \delta \mathcal{S}\right)-\frac{2}{3} \Delta \delta \mathcal{S}\right] \\
& -\frac{8 \pi G}{3}(\rho+p) \Delta \chi-4 \pi G\left(p^{\prime}-c_{s}^{2} \rho^{\prime}\right) \chi^{\prime}
\end{aligned}
$$

Here the part which does not contain $\chi$ is the standard GR dynamical equation for the potential $\Phi$, while the part containing $\chi$ is the modification due to non-metricity. In obtaining (6.25), we used the background field equations. Let us also give an expression for the constraint (6.18) in terms of the potential $\Phi$. Again using the background equations, we obtain

$$
4 \pi G a^{2} \delta \rho=\Delta \Phi-3 \mathcal{H} \Phi^{\prime}-3 \mathcal{H}^{2} \Phi+6 \pi G a^{2}\left[\Delta \delta \mathcal{S}-3 \mathcal{H}\left(\delta \mathcal{S}^{\prime}+\mathcal{H} \delta \mathcal{S}\right)\right]-4 \pi G \rho^{\prime} \chi^{\prime} .
$$

The last term here is the contribution due to non-metricity. One can also give an equation for the velocity perturbation in terms of the new potential:

$$
4 \pi G a \delta u=\Phi^{\prime}+\mathcal{H} \Phi+6 \pi G a^{2}\left(\delta \mathcal{S}^{\prime}+\mathcal{H} \delta \mathcal{S}\right)-4 \pi G(\rho+p) \chi^{\prime} .
$$

Finally, we will also need an expression for equation (6.19) in terms of the new potential $\Phi:$

$$
\begin{aligned}
4 \pi G a^{2} \delta p=\Phi^{\prime \prime}+3 \mathcal{H} \Phi^{\prime}+\left(2 \mathcal{H}^{\prime}+\mathcal{H}^{2}\right) \Phi+6 \pi G a^{2} & {\left[\delta \mathcal{S}^{\prime \prime}+5 \mathcal{H}\left(\delta \mathcal{S}^{\prime}+\mathcal{H} \delta \mathcal{S}\right)-\frac{2}{3} \Delta \delta \mathcal{S}\right] } \\
& -\frac{8 \pi G}{3}(\rho+p) \Delta \chi-4 \pi G p^{\prime} \chi^{\prime}
\end{aligned}
$$




\subsection{Applicability limits of the theory}

As we have discussed above, our classical theory is applicable in the case of small deviations from the "metric" behavior, which is quantitatively expressed as (2.25). Since we have (2.24) and, in the linear theory, according to (5.6),

$$
H^{i j} \approx \delta B^{i j}=\frac{1}{a^{2}}\left(\chi_{, i j}-\frac{1}{3} \delta_{i j} \Delta \chi\right)
$$

we have the following condition:

$$
\frac{k^{2}}{a^{2}}|\chi|=\frac{k^{2}}{a^{2}}\left|\frac{\Phi}{4 \pi G \kappa+2 \mathcal{H}^{2} / a^{2}}\right| \ll 1 .
$$

This condition is not stronger than the usual condition of smallness of density contrast:

$$
\left|\frac{\delta \rho+3(\mathcal{H} / a) \delta u-\frac{3}{2} \Delta \mathcal{S}}{\rho}\right| \ll 1 \quad \Longleftrightarrow \quad \frac{2 k^{2}}{3 \mathcal{H}^{2}-\Lambda_{0} a^{2}}|\Phi| \ll 1,
$$

provided the denominator $4 \pi G \kappa+2 H^{2}$ in (6.30) does not approach zero sufficiently closely. We have to assume that this never happens and that always

$$
2 \pi G \kappa+\frac{\mathcal{H}^{2}}{a^{2}}>0
$$

Note that this condition is also necessary in order that the change of variable (6.23) be nondegenerate. Recalling the definition (4.4) of $\kappa$, we obtain a constraint on the parameter $\beta$ or $g$ by considering a dust-dominated universe: $\beta>-4 / 3$, or $g>-1$. Considering the inflationary universe with $p \approx-\rho$, we get a stronger condition $\beta>-1 / 3$, or $g>0$. Finally, considering the "stiff" matter with $p=\rho$ (which is realized, e.g., by the kineticallydominated regime of a scalar field), we have the constraint $\beta<2 / 3$, or $g<1$. Thus, the physical values of $g$ or, respectively, $\beta=g-1 / 3$ lie in the domain

$$
0<g<1, \quad-\frac{1}{3}<\beta<\frac{2}{3} .
$$

In a similar way, we obtain a constraint on the parameter $\gamma$ by considering a universe which is dominated by the cosmological constant: $\gamma>-4 / 3$. An even stronger condition can be obtained by considering a radiation-dominated universe. We then have:

$$
\gamma>0
$$

We, therefore, assume that inequalities $(6.33)$ and $(\sqrt[6.34]{ })$ are satisfied by a large margin. In particular, the quantity $\beta$ cannot be much larger than unity by absolute value.

\section{Evolution of scalar perturbations}

\subsection{Inflation}

The primordial spectrum of perturbations is obtained in the inflationary paradigm. Therefore, we start with application of our theory to the simplest model of inflation based on a 
single inflaton field $\varphi$. We assume that the inflaton has the usual coupling to the metric defined by the $B$-field. Then its action can be written as

$$
S[\varphi]=\int d^{4} x \sqrt{-\operatorname{det}\left(g_{\mu \nu}\right)}\left[g^{\mu \nu} \partial_{\mu} \varphi \partial_{\nu} \varphi-V(\varphi)\right],
$$

where $g_{\mu \nu}$ is the metric defined by $B$.

The background field equations for $\varphi$ take the usual form

$$
\mathcal{H}^{2}=\frac{8 \pi G}{3}\left[\frac{1}{2} \varphi^{\prime 2}+a^{2} V(\varphi)\right]+\frac{\Lambda_{0} a^{2}}{3}, \quad \mathcal{H}^{2}-\mathcal{H}^{\prime}=4 \pi G \varphi^{\prime 2}
$$

where, as before, the prime denotes the derivative with respect to the conformal time.

The equations for perturbations can be obtained from the general equations $(6.18)$ and (6.20) by substituting appropriate expressions for $\delta \rho$ and $\delta u$ :

$$
\delta \rho \rightarrow\left(\frac{\varphi^{\prime}}{a}\right)^{2}\left[\left(\frac{\delta \varphi}{\varphi^{\prime}}\right)^{\prime}-2 \mathcal{H} \frac{\delta \varphi}{\varphi^{\prime}}-\Phi_{\mathrm{GR}}\right], \quad \delta u \rightarrow \frac{\varphi^{\prime}}{a} \delta \varphi .
$$

From these expressions, we find

$$
\delta \rho+\frac{3 \mathcal{H}}{a} \delta u=\left(\frac{\varphi^{\prime}}{a}\right)^{2}\left[\left(\frac{\delta \varphi}{\varphi^{\prime}}\right)^{\prime}+\mathcal{H} \frac{\delta \varphi}{\varphi^{\prime}}-\Phi_{\mathrm{GR}}\right] .
$$

To obtain a dynamical equation for perturbations, it remains to express this quantity in terms of the potential $\Phi$ using (6.24). For this purpose, we first use equation (6.13) to express $\Phi_{\mathrm{GR}}$ in terms of the potential $\Psi_{\mathrm{GR}}$ :

$$
\mathcal{H} \Phi_{\mathrm{GR}}=\left(\mathcal{H}^{2}-\mathcal{H}^{\prime}\right) \frac{\delta \varphi}{\varphi^{\prime}}+\frac{1}{3 a^{2}} \Delta \chi^{\prime}-\Psi_{\mathrm{GR}}^{\prime},
$$

where we have used expression (7.3) for $\delta u$ as well as the background field equations (7.2). Substituting (7.5) into (7.4) and once again using the background field equations, we can write equation (6.24) as follows:

$$
\frac{\mathcal{H}^{2}-\mathcal{H}^{\prime}}{\mathcal{H}}\left(\mathcal{H} \frac{\delta \varphi}{\varphi^{\prime}}+\Psi_{\mathrm{GR}}\right)^{\prime}-\frac{\mathcal{H}^{2}-\mathcal{H}^{\prime}}{3 a^{2} \mathcal{H}} \Delta \chi^{\prime}=\Delta \Phi,
$$

where, as before, $\Phi$ is given by (6.23).

Now we use expressions (6.20) and (6.10), respectively, for $\delta u$ and $\Psi_{\mathrm{GR}}$ in terms of $\chi$ to transform the expression in the brackets of (7.6) into the following form:

$$
\mathcal{H} \frac{\delta \varphi}{\varphi^{\prime}}+\Psi_{\mathrm{GR}}=\frac{\mathcal{H}^{2} / a^{2}}{\mathcal{H}^{2}-\mathcal{H}^{\prime}}\left(\frac{a^{2} \Phi}{\mathcal{H}}\right)^{\prime}+\frac{1}{3 a^{2}} \Delta \chi
$$

where we have used the definition (6.23) of the potential $\Phi$. Combining equations (7.6) and (7.7), we see that the term containing $\Delta \chi^{\prime}$ cancels. The remaining term with $\Delta \chi$ can be converted to a term with $\Delta \Phi$ by using the relation (6.23) between $\chi$ and $\Phi$. The final equation for the potential $\Phi$ is

$$
\frac{\mathcal{H}^{2}-\mathcal{H}^{\prime}}{\mathcal{H}}\left[\frac{\mathcal{H}^{2} / a^{2}}{\mathcal{H}^{2}-\mathcal{H}^{\prime}}\left(\frac{a^{2} \Phi}{\mathcal{H}}\right)^{\prime}\right]^{\prime}=\left[1+\frac{\mathcal{H}^{2}-\mathcal{H}^{\prime}}{3\left(\mathcal{H}^{2}+6 \pi G a^{2} \kappa\right)}\right] \Delta \Phi .
$$


Let us also present it in terms of the derivatives with respect to the physical time:

$$
\frac{\dot{H}}{H} \partial_{t}\left[\frac{H^{2}}{a \dot{H}} \partial_{t}\left(\frac{a \Phi}{H}\right)\right]=\left[1-\frac{\dot{H}}{3\left(H^{2}+6 \pi G \kappa\right)}\right] \frac{\Delta \Phi}{a^{2}} .
$$

Apart from the term on the right-hand side proportional to $\dot{H}$, this is the usual equation for the evolution of the relativistic potential during inflation. Note that the denominator $H^{2}+6 \pi G \kappa$ in the last term on the right-hand side of $(7.9)$ is positive by virtue of constraints (6.33).

The quantity $6 \pi G \kappa$ in the case under consideration is given by the expression

$$
6 \pi G \kappa=2 \pi G(3 g-1)(\rho-3 p)+\frac{3}{4 \ell^{2}}-\Lambda_{0}=(3 g-1)\left(3 H^{2}+\frac{3}{2} \dot{H}\right)+\frac{3}{4 \ell^{2}}-3 g \Lambda_{0} .
$$

As we have already noted in Sec. 2.6, the equations for perturbations, in general, are not invariant under the simultaneous change

$$
\Lambda_{0} \rightarrow \Lambda_{0}-\lambda, \quad V(\varphi)+\frac{\lambda}{8 \pi G},
$$

which would be the case in general relativity. However, they are invariant after an additional change

$$
\frac{1}{\ell^{2}} \rightarrow \frac{1}{\ell^{2}}-4 g \lambda
$$

In particular, such a transformation will arise in the case where the parameter $\ell^{2}$ is fixed to be $\ell^{2}=1 / 4 g \Lambda_{0}$.

During the inflationary epoch, we have $|\dot{H}| \ll H^{2}$, and one can neglect the term containing $\dot{H}$ on the right-hand side of (7.9). Thus, we can argue that the evolution of inflation-generated perturbations is unchanged in the theory under consideration, and we can take the standard flat spectrum for the potential $\Phi$ as initial conditions. This conclusion for the generated spectrum can be justified as follows. Equations (6.24) and (7.4) imply the relation

$$
\Delta \Phi+4 \pi G \varphi^{\prime 2} \Phi_{\mathrm{GR}}=\frac{4 \pi G \varphi^{\prime 2}}{a}\left(\frac{a \delta \varphi}{\varphi^{\prime}}\right)^{\prime} .
$$

On spatial scales smaller than the Hubble scales, with the comoving wavenumber $k$ satisfying $k^{2} \gg \mathcal{H}^{2}$, we can neglect the self-gravity of the scalar field and quantize the inflaton perturbation $\delta \varphi$ on the background of a homogeneous and isotropic inflationary universe, obtaining the standard spectrum for the modes $\delta \varphi_{\mathbf{k}}$. Then we can use equation (7.13) to match the quantities $\Phi$ and $\delta \varphi$ on small (sub-Hubble) spatial scales during inflation. In doing this, the term $4 \pi G \varphi^{2} \Phi_{\mathrm{GR}}$ in 7.13 can be neglected (just as it is the case with a similar term in GR) since, according to $(\sqrt[6.11]{)})$, it is estimated as

$$
4 \pi G \varphi^{\prime 2} \Phi_{\mathrm{GR}}=4 \pi G \varphi^{\prime 2}\left[\Phi+\mathcal{O}\left(\mathcal{H}^{-1}\right) \Phi^{\prime}+\mathcal{O}\left(\mathcal{H}^{-2}\right) \Phi^{\prime \prime}+\mathcal{O}\left(\mathcal{H}^{-2}\right) \Delta \Phi\right]
$$

and is small in view of the condition $k^{2} \gg \mathcal{H}^{2} \gg 4 \pi G \varphi^{\prime 2}$. Thus, the quantity $\Phi$ on small scales will acquire the standard amplitude with scale-invariant spectrum and then will 
evolve according to equation (7.9), which, as we noted, differs negligibly from the standard inflationary equation for the relativistic potential.

In the regime of very long wavelengths, we can neglect all terms with Laplacians. In this case, equation (7.13) implies the relation

$$
\Phi_{\mathrm{GR}} \approx \frac{1}{a}\left(\frac{a \delta \varphi}{\varphi^{\prime}}\right)^{\prime}=\left(\frac{\delta \varphi}{\varphi^{\prime}}\right)^{\prime}+\frac{\mathcal{H} \delta \varphi}{\varphi^{\prime}} .
$$

Substituting this into (7.3), we get

$$
\delta \rho \approx-3 \mathcal{H}\left(\frac{\varphi^{\prime}}{a}\right)^{2} \frac{\delta \varphi}{\varphi^{\prime}}=-\frac{3 \mathcal{H}}{a^{2}} \varphi^{\prime} \delta \varphi .
$$

Equations (6.10) and (6.11) with Laplacians neglected give

$$
\Psi_{\mathrm{GR}} \approx \Phi+\frac{\mathcal{H}}{a^{2}} \chi^{\prime}, \quad \Phi_{\mathrm{GR}} \approx \Phi-\frac{1}{a^{2}} \chi^{\prime \prime}+\frac{\mathcal{H}}{a^{2}} \chi^{\prime} .
$$

Then, according to (7.2) and (7.5), we have

$$
4 \pi G \varphi^{\prime} \delta \varphi \approx \mathcal{H} \Phi_{\mathrm{GR}}+\Psi_{\mathrm{GR}}^{\prime} \approx \mathcal{H} \Phi+\Phi^{\prime}-4 \pi G \varphi^{\prime 2} \chi^{\prime} \approx \mathcal{H} \Phi
$$

where, in the last approximation, we have neglected the two terms $\Phi^{\prime}-4 \pi G \varphi^{\prime 2} \chi^{\prime}$ compared

to $\mathcal{H} \Phi$, which is legitimate during inflation. Comparing $(7.16)$ and $(\mathbf{7 . 1 8})$, we get the usual relation

$$
\frac{\delta \rho}{\rho} \approx-2 \Phi,
$$

valid on super-Hubble spatial scales during inflation. Note that, in the same inflationary approximation, we have $\Phi_{\mathrm{GR}} \approx \Psi_{\mathrm{GR}} \approx \Phi$ on super-Hubble spatial scales.

\subsection{Evolution with a generic equation of state}

In this subsection, we would like to demonstrate that, on asymptotically large spatial scales, the relativistic potential $\Phi$ defined in (6.23) behaves just as in general relativity, including the cases where the universe experiences slow or rapid transitions between epochs with different effective equations of state. To see this, consider equation 6.25) in the case of vanishing shear $\mathcal{S}$ for a system described by a generic equation of state $p(\rho)$, which can interpolate between different regimes. Note that this assumption implies vanishing of the entropy perturbation, $\delta \mathcal{E}=0$, as well as of the last term in equation (6.25), which equation then takes the form

$$
\Phi^{\prime \prime}-c_{s}^{2} \Delta \Phi+3 \mathcal{H}\left(1+c_{s}^{2}\right) \Phi^{\prime}+\left[2 \mathcal{H}^{\prime}+\mathcal{H}^{2}\left(1+3 c_{s}^{2}\right)\right] \Phi-\frac{8 \pi G}{3}(\rho+p) \Delta \chi=0 .
$$

By the standard change of function (see [11, Sec. 7.3])

$$
\Phi=u \exp \left[-\frac{3}{2} \int\left(1+c_{s}^{2}\right) \mathcal{H} d \eta\right] \propto(\rho+p)^{1 / 2} u
$$

equation $(7.20)$ is transformed to

$$
u^{\prime \prime}-c_{\mathrm{eff}}^{2} \Delta u-\frac{\vartheta^{\prime \prime}}{\vartheta} u=0,
$$


where

$$
\begin{gathered}
\vartheta \equiv \frac{1}{a}\left[\frac{2}{3}\left(1-\frac{\mathcal{H}^{\prime}}{\mathcal{H}^{2}}\right)\right]^{-1 / 2}=\frac{1}{a}\left(\frac{\rho+\rho_{\Lambda}}{\rho+p}\right)^{1 / 2}, \\
c_{\mathrm{eff}}^{2}=c_{s}^{2}+\frac{2(\rho+p)}{3 \beta(\rho-3 p)+3 \gamma \rho_{\Lambda}+4 \rho},
\end{gathered}
$$

and we have used definition (6.23) and neglected the cosmological constant contribution in the denominator.

The long-wave solution of $(7.22)$ is obtained when the term with Laplacian is neglected:

$$
u(\eta) \approx C \vartheta(\eta) \int_{\eta_{0}}^{\eta} \frac{d \eta^{\prime}}{\vartheta^{2}\left(\eta^{\prime}\right)},
$$

where $C$ and $\eta_{0}$ are constants of integration. In this regime, the quantity

$$
\zeta=\vartheta^{2}\left(\frac{u}{\vartheta}\right)^{\prime} \propto \Phi+\frac{2}{3} \frac{\rho+\rho_{\Lambda}}{\rho+p}\left(\Phi+\frac{1}{\mathcal{H}} \Phi^{\prime}\right)
$$

is conserved.

Equation (7.22) can be recast in the form

$$
\left[\vartheta^{2}\left(\frac{u}{\vartheta}\right)^{\prime}\right]^{\prime}=c_{\mathrm{eff}}^{2} \vartheta^{2} \Delta\left(\frac{u}{\vartheta}\right)
$$

which, in particular, shows that the quantities

$$
\frac{u}{\vartheta}=a\left(\frac{\rho+p}{\rho+\rho_{\Lambda}}\right)^{1 / 2} u \propto \frac{a^{2}}{\mathcal{H}} \Phi
$$

and

$$
\vartheta^{2}\left(\frac{u}{\vartheta}\right)^{\prime}-\frac{\vartheta^{2}}{3 \mathcal{H}} \Delta\left(\frac{u}{\vartheta}\right) \propto \Phi+\frac{2}{3} \frac{\rho+\rho_{\Lambda}}{\rho+p}\left(\Phi+\frac{1}{\mathcal{H}} \Phi^{\prime}-\frac{1}{3 \mathcal{H}^{2}} \Delta \Phi\right)
$$

should remain continuous during a rapid transition between different equations of state. Since $a$ and $\mathcal{H}$ are obviously continuous in this case, it follows that $\Phi$ must also be continuous.

The relations derived in this subsection imply that the inflationary and post-inflationary evolution of the potential $\Phi$ on large spatial scales will reproduce those of general relativity. The only modification as compared with GR occurs on small spatial scales, where oscillations in (7.22) proceed with effective speed of sound (7.24), different from $c_{s}^{2}$. During some periods in the cosmological evolution, this difference can be small and lead to negligible effect in (7.22). In this case, characterized by

$$
\frac{2(\rho+p)}{3 \beta(\rho-3 p)+3 \gamma \rho_{\Lambda}+4 \rho} \ll c_{s}^{2},
$$

we will say that perturbations evolve in the regime of general relativity (GR) on all scales. In the opposite case, when the difference between $c_{\text {eff }}^{2}$ and $c_{s}^{2}$ is essential, we will say that they evolve in the regime of modified gravity. 
From (6.10) and (6.11), one can obtain relations between the relativistic potentials on small scales:

$$
\begin{aligned}
& \Psi_{\mathrm{GR}}=\Phi+\frac{1}{a^{2}}\left(\frac{1}{3} \Delta \chi+\mathcal{H} \chi^{\prime}\right) \approx\left[1-\frac{k^{2}}{12 \pi G a^{2} \kappa+6 \mathcal{H}^{2}}\right] \Phi, \quad k^{2} \gg \mathcal{H}^{2}, \\
& \Phi_{\mathrm{GR}}=\Phi+\frac{1}{a^{2}}\left(\frac{2}{3} \Delta \chi-\chi^{\prime \prime}+\mathcal{H} \chi^{\prime}\right) \approx\left[1+\frac{\left(3 c_{\mathrm{eff}}^{2}-2\right) k^{2}}{12 \pi G a^{2} \kappa+6 \mathcal{H}^{2}}\right] \Phi, \quad k^{2} \gg \mathcal{H}^{2} .
\end{aligned}
$$

In the regime of general relativity, where $2 \pi G \kappa \gg \mathcal{H}^{2} / a^{2}$, we have

$$
\Psi_{\mathrm{GR}} \approx\left[1-\frac{2 k^{2} \ell^{2}}{3 a^{2}}\right] \Phi, \quad \Phi_{\mathrm{GR}} \approx\left[1+2\left(c_{\mathrm{eff}}^{2}-\frac{2}{3}\right) \frac{k^{2} \ell^{2}}{a^{2}}\right] \Phi, \quad k^{2} \gg \mathcal{H}^{2} .
$$

In deriving the above relations, we have taken into account that, in the high-frequency regime under consideration, $\chi^{\prime \prime} \approx c_{\text {eff }}^{2} \Delta \chi$, so that the term proportional to $\chi^{\prime \prime}$ in (6.11) gives a contribution of the same order as $\Delta \chi$. We see that, even in the GR regime, the usual GR relation $\Phi_{\mathrm{GR}}=\Psi_{\mathrm{GR}}$ is violated for physical wave numbers $k / a>1 / \ell$, i.e., on scales smaller than the scale of deformation $\ell$.

\subsection{Radiation domination}

The evolution of scalar perturbations in a universe filled with fluid are most easily analyzed using the system of equations (6.25), (6.26). For simplicity, we set the shear to zero and restrict ourselves to adiabatic perturbations, $\delta \mathcal{E}=0$.

At the stage of radiation domination, we have $a=a_{0} \eta$, so that $\mathcal{H}=1 / \eta, \mathcal{H}^{\prime}=-\mathcal{H}^{2}$, and $\mathcal{H}^{\prime \prime}=2 \mathcal{H}^{3}$. The speed of sound at this stage is $c_{s}^{2}=1 / 3$. Equation (6.25) then simplifies to

$$
\Phi^{\prime \prime}+\frac{4}{\eta} \Phi^{\prime}-c_{\mathrm{eff}}^{2} \Delta \Phi=0
$$

where

$$
c_{\mathrm{eff}}^{2}=\frac{\gamma \rho_{\Lambda}+4 \rho}{3 \gamma \rho_{\Lambda}+4 \rho}
$$

and, in using equation (6.23), we have taken into account that $\rho=3 p$, hence, $\kappa=\gamma \rho_{\Lambda}=$ const. Equation (7.34) has the form of the usual equation for a radiation-dominated universe apart from the fact that the effective speed of sound became time-dependent.

In a very early universe, $\rho \gg \gamma \rho_{\Lambda}$, and the effective speed of sound (7.35) becomes equal to the speed of light. This is the modified-gravity regime. In the course of time, if the quantity $\gamma \rho_{\Lambda}$ becomes dominant over $\rho$, the effective speed of sound (7.35) turns to its standard value $1 / \sqrt{3}$. This is the GR regime. As usual, the limit of GR is obtained by sending $\gamma \rightarrow \infty$.

From the constraint (6.26), we find the expression for energy-density perturbations during this stage:

$$
4 \pi G a^{2} \delta \rho=\Delta \Phi-3 \mathcal{H} \Phi^{\prime}\left(\frac{3 \gamma \rho_{\Lambda}}{3 \gamma \rho_{\Lambda}+4 \rho}\right)-3 \mathcal{H}^{2} \Phi\left[1-\frac{4(4 \rho)^{2}}{\left(3 \gamma \rho_{\Lambda}+4 \rho\right)^{2}}\right],
$$


where we have used the fact that $\kappa=\gamma \rho_{\Lambda}=$ const during radiation domination and that $\rho^{\prime}=-4 \mathcal{H} \rho$. Again, as $\gamma \rightarrow \infty$, this equation takes the standard GR form.

As usual, the qualitative behavior of solutions of (7.34) is easy to understand in the two asymptotic regimes: $k \eta \ll 1$, corresponding to modes with wavelength larger than the Hubble radius, and $k \eta \gg 1$, corresponding to modes that already entered the Hubble radius. In the first case, the term $\Delta \Phi$ can be neglected, and $\Phi=$ const is a non-decreasing solution. In this case, the last term in equation (7.36) is dominant and gives $a^{4} \delta \rho \propto \Phi$ with coefficient of proportionality being a function of time. Note that the usual generalrelativity relation $\delta \rho / \rho=-2 \Phi$ is no longer valid in our theory. Indeed, this will hold only in the GR regime, where $\rho \ll \gamma \rho_{\Lambda}$, while in the early universe we may have $\rho \gg \gamma \rho_{\Lambda}$. Thus, for $k \eta \ll 1$, we have

$$
\frac{\delta \rho}{\rho} \approx\left\{\begin{aligned}
6 \Phi, \rho & \gg \gamma \rho_{\Lambda} \text { (modified-gravity regime) } \\
-2 \Phi, \rho & \ll \gamma \rho_{\Lambda} \text { (GR regime) }
\end{aligned}\right.
$$

As a consequence, the long-wave gauge-invariant energy-density perturbation $\delta \rho / \rho$ passes through zero for $\gamma \rho_{\Lambda} \sim \rho$, which is an interesting phenomenon connected with the gauge choice in this model.

Note that the standard relation

$$
\frac{\delta \rho}{\rho} \approx-2 \Phi_{\mathrm{GR}}
$$

remains to be true in both regimes, in view of relation (7.17) between the potentials $\Phi$ and $\Phi_{\mathrm{GR}}$ valid in the long-wave approximation. Since it is the quantity $\Phi$ which remains constant on large scales during radiation domination, both $\delta \rho / \rho$ and $\Phi_{\mathrm{GR}}$ change in the transition between the regimes of modified gravity and GR. Relations (7.17) in this case read

$$
\begin{aligned}
& \Psi_{\mathrm{GR}} \approx\left[1+\frac{2(4 \rho)^{2}}{\left(3 \gamma \rho_{\Lambda}+4 \rho\right)^{2}}\right] \Phi \approx\left\{\begin{array}{c}
3 \Phi, \rho \gg \gamma \rho_{\Lambda} \text { (modified-gravity regime) }, \\
\Phi, \rho \ll \gamma \rho_{\Lambda} \text { (GR regime) } .
\end{array}\right.
\end{aligned}
$$

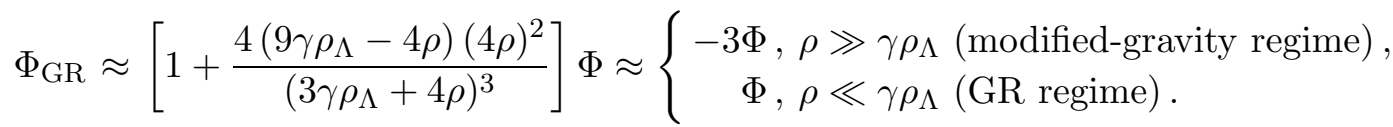

For modes with $k \eta \gg 1$, the term $\Delta \Phi$ in $(7.34)$ is important. The dominant solution of (7.34) oscillates with frequency determined by the effective speed of sound (7.35) and with a decaying amplitude $\propto \eta^{-2} c_{\mathrm{eff}}^{-1 / 2} \propto a^{-2} c_{\mathrm{eff}}^{-1 / 2}$. In this case, the first term on the right-hand side of (7.36) is dominant, and the relation between $\delta \rho / \rho$ and the potential $\Phi$ is the same as in GR. The energy-density perturbation $\delta \rho / \rho$ is now an oscillating function with frequency determined by $(7.35)$ and with amplitude $\propto c_{\text {eff }}^{-1 / 2}$, which is constant both in the modified and GR epochs and growing during the transition. The relativistic potentials on such small scales are described by the general equations (7.31)-(7.33).

Thus, as in GR, perturbations do not grow during radiation domination. This is true both for the modified-gravity epoch in early universe and for the GR epoch at late times. However, the relation between the energy density fluctuation $\delta \rho / \rho$ and the main relativistic 
potential $\Phi$ on the super-Hubble scales is different at these epochs, as shown by (7.37). The effective speed of sound (7.35), which determines oscillations on sub-Hubble scales, is also different at different epochs. During the transition occurring for $\gamma \rho_{\Lambda} \sim \rho$, if it is realized at the radiation-dominated stage, the long-wave gauge-invariant energy-density perturbation $\delta \rho / \rho$ passes through zero.

Perturbations at the radiation-dominated stage are studied in greater detail in Sec. 8 below.

\subsection{Matter domination}

At this stage, we have $p=\delta p=0, a(\eta) \propto \eta^{2}$, and equation (6.25) simplifies to

$$
\Phi^{\prime \prime}+\frac{6}{\eta} \Phi^{\prime}-c_{\mathrm{eff}}^{2} \Delta \Phi=0
$$

where

$$
c_{\mathrm{eff}}^{2}=\frac{2 \rho}{3 \kappa+4 \rho} .
$$

In this case, $\kappa=\beta \rho+\gamma \rho_{\Lambda}$ is not constant [ $\beta$ and $\gamma$ are defined in (4.5)]. On sufficiently large spatial scales, $k \ll \mathcal{H} / c_{\text {eff }}$, we have $\Phi \approx$ const while, on small scales, $k \gg \mathcal{H} / c_{\text {eff }}$, the potential $\Phi$ oscillates with decreasing amplitude $\propto \eta^{-3} c_{\text {eff }}^{-1 / 2} \propto a^{-3 / 2} c_{\text {eff }}^{-1 / 2}$ and with effective speed of sound given by (7.42). This behavior is quite different from the case of general relativity, where the corresponding relativistic potential remains constant at all spatial scales.

To determine the behavior of the density contrast, consider the quantity

$$
\delta_{m}:=\frac{\delta \rho+3(\mathcal{H} / a) \delta u}{\rho},
$$

which is related to $\Phi$ through (6.24):

$$
4 \pi G a^{2} \rho \delta_{m}=\Delta \Phi
$$

We see that, on large spatial scales, $k \ll \mathcal{H} / c_{\text {eff }}$, the density contrast grows like in GR: $\delta_{m} \propto a$, while, on small spatial scales, $k \gg \mathcal{H} / c_{\text {eff }}$, it oscillates with a slightly increasing amplitude $\propto a^{-1 / 2} c_{\text {eff }}^{-1 / 2} \propto a^{1 / 4}$ and frequency determined by the effective speed of sound in (7.42).

It is useful to obtain a closed equation describing the evolution of $\delta_{m}$. Using the conservation equations (6.16) and (6.17) as well as our assumption $p=\delta p=0$, we get

$$
\rho \delta_{m}^{\prime}=3 \rho\left(\Psi_{\mathrm{GR}}^{\prime}+\mathcal{H} \Phi_{\mathrm{GR}}\right)+\frac{1}{a} \Delta \delta u+\frac{3}{a}\left(\mathcal{H}^{\prime}-\mathcal{H}^{2}\right) \delta u .
$$

Taking into account equation (6.13) and the background equation $\mathcal{H}^{2}-\mathcal{H}^{\prime}=4 \pi G a^{2}(\rho+p)$, we see that the terms proportional to $\delta u$ cancel, so that

$$
\delta_{m}^{\prime}=\frac{1}{a^{2}} \Delta \chi^{\prime}+\frac{1}{a \rho} \Delta \delta u
$$


Taking the second derivative of $\delta_{m}$, we obtain

$$
\delta_{m}^{\prime \prime}=\frac{1}{a^{2}}\left(\Delta \chi^{\prime \prime}-2 \mathcal{H} \Delta \chi^{\prime}\right)+\Delta \Phi_{\mathrm{GR}}-\frac{\mathcal{H}}{a \rho} \Delta \delta u,
$$

where we have used the conservation equation (6.17) once again. The following identity is a direct consequence of $(6.9)$ and $(6.10)$ :

$$
\Delta \Phi_{\mathrm{GR}}+\frac{1}{a^{2}} \Delta\left(\chi^{\prime \prime}-\mathcal{H} \chi^{\prime}\right)=\frac{2}{3 a^{2}} \Delta^{2} \chi+\left(4 \pi G \kappa+\frac{2 \mathcal{H}^{2}}{a^{2}}\right) \Delta \chi .
$$

Combining (7.46)-(7.48) and using the relation (7.44) between $\delta_{m}$ and $\Delta \chi$, we have

$$
\delta_{m}^{\prime \prime}+\mathcal{H} \delta_{m}^{\prime}-\frac{2}{3 a^{2}} \Delta^{2} \chi=4 \pi G a^{2} \rho \delta_{m}
$$

Finally, using the relation (6.23) between $\Phi$ and $\chi$ as well as equation (7.44), we obtain

$$
\delta_{m}^{\prime \prime}+\mathcal{H} \delta_{m}^{\prime}-\frac{2 \rho}{3 \kappa+4 \rho} \Delta \delta_{m}=4 \pi G a^{2} \rho \delta_{m}
$$

It is illuminating to rewrite the above equation in terms of the physical time:

$$
\ddot{\delta}_{m}+2 H \dot{\delta}_{m}-\frac{2 \rho}{3 \kappa+4 \rho} \frac{\Delta \delta_{m}}{a^{2}}=4 \pi G \rho \delta_{m}
$$

where $H=\dot{a} / a$ is the physical Hubble parameter. Equation (7.51) allows for a straightforward passage to the GR limit: one just has to send $\kappa \rightarrow \infty$. For finite $\kappa$, we get an extra term that leads to oscillations on sub-Hubble scales, an effect absent in general relativity.

In view of the definition $\kappa=\beta \rho+\gamma \rho_{\Lambda}$, we can write:

$$
c_{\text {eff }}^{2}=\frac{2 / 3}{\beta+4 / 3+\gamma \rho_{\Lambda} / \rho} .
$$

Thus, at the early epoch with $\rho \gg \gamma \rho_{\Lambda}$ (if this condition is realized during matter domination), we have oscillations with effective speed of sound $c_{\text {eff }}^{2}=2 /(3 \beta+4)$. If $\gamma \gg 1$, then, as the energy density decreases with the expansion, one may enter into the regime $\rho_{\Lambda} \ll \rho \ll \gamma \rho_{\Lambda}$ in which the usual GR behavior is recovered in a broad range of spatial scales. Note that the physical spatial scale on which oscillations take place $k / a>\mathcal{H} / c_{\text {eff }} a \sim a^{-3 / 2}\left(\gamma \rho_{\Lambda} / \rho\right)^{1 / 2} \sim 1 / \ell$ remains constant during the matter domination era.

\subsection{Lambda domination}

At later epochs in its evolution, the universe is dominated by the cosmological constant. It is thus necessary to consider the evolution of matter perturbations in such a universe. The scale factor in a Lambda-dominated universe is given by

$$
a(\eta)=-\frac{1}{\eta} \sqrt{\frac{3}{\Lambda_{0}}},
$$


so that $\mathcal{H}=-1 / \eta$ and $\mathcal{H}^{\prime}=1 / \eta^{2}=\mathcal{H}^{2}$. Equation (6.25) in this approximation reads

$$
\Phi^{\prime \prime}-\frac{3}{\eta} \Phi^{\prime}+\frac{3}{\eta^{2}} \Phi-c_{\mathrm{eff}}^{2} \Delta \Phi=0
$$

with the effective speed of sound

$$
c_{\text {eff }}^{2}=\frac{4 \pi G \rho}{6 \pi G \kappa+3 \mathcal{H}^{2} / a^{2}}=\frac{2 \rho}{(3 \beta+4) \rho+(3 \gamma+4) \rho_{\Lambda}} \sim \frac{2}{3 \gamma+4}\left(\rho / \rho_{\Lambda}\right) \ll 1,
$$

where we have taken into account that $\mathcal{H}^{2} / a^{2}=\Lambda_{0} / 3+8 \pi G \rho / 3, \kappa=\beta \rho+\gamma \rho_{\Lambda}$, and the $\Lambda$-domination condition $\rho_{\Lambda} \gg \rho$. Equation (7.54) in this case differs from the generalrelativistic analog only by the term with $c_{\text {eff }}^{2} \Delta \Phi$.

On sufficiently large scales, $k \ll \mathcal{H} / c_{\text {eff }}$, the evolution proceeds as in the case of general relativity, with the dominant mode

$$
\Phi \propto \eta \propto a^{-1}
$$

The evolution of the density contrast is given by equation (6.26):

$$
4 \pi G a^{2} \delta \rho=\Delta \Phi-4 \pi G \rho^{\prime} \chi^{\prime}=\Delta \Phi-\frac{9}{2} c_{\mathrm{eff}}^{2} \mathcal{H}^{2} \Phi .
$$

One can see that the evolution is the same as in general relativity, $\delta \rho / \rho \propto a \Phi=$ const in the range of scales $c_{\text {eff }} \mathcal{H} \ll k \ll \mathcal{H} / c_{\text {eff }}$. However, the lower boundary in this case $\left(c_{\text {eff }}^{2} \ll 1\right)$ corresponds to the scales well beyond the Hubble radius, which are unobserved in our universe. Thus, we may conclude that the long-wave perturbations, with $k \ll \mathcal{H} / c_{\text {eff }}$, evolve in the GR regime.

On small scales, $k \gg \mathcal{H} / c_{\text {eff }}$, equation $(7.54)$ describes oscillations with adiabatically decreasing amplitude $\propto a^{-3 / 2} c_{\text {eff }}^{-1 / 2} \propto a^{-3 / 4}$ (in the universe with pressureless matter). In this case, for $c_{\text {eff }}^{2} \ll 1$, we have

$$
4 \pi G a^{2} \delta \rho \approx \Delta \Phi
$$

so that the density contrast $\delta \rho / \rho$ oscillates with amplitude $\propto a^{-1 / 2} c_{\text {eff }}^{-1 / 2} \propto a^{1 / 4}$, similar to what happens in the regime of matter domination.

The threshold comoving frequency which distinguishes between these two types of behavior is growing as $k_{\Lambda}:=\mathcal{H} / c_{\text {eff }} \propto a^{5 / 2}$, and so the physical threshold scale $a / k_{\Lambda}$ decreases from its value $\sim \ell$ at the matter-dominated stage as $a / k_{\Lambda} \propto a^{-3 / 2}$.

\section{Effects of the modification of gravity on the power spectrum}

In this section, we give a qualitative analysis of the effect of modification of gravity on the linear evolution of matter and radiation density perturbations in the adiabatic mode.

First of all, as we have seen in Sec. 7.1, the spectrum on super-Hubble scales generated during inflation is practically the same as in the usual inflationary theory based on general relativity. The subsequent evolution of the spectrum depends on the epoch at which the condition $\rho=\gamma \rho_{\Lambda}$ is reached, i.e., it depends on the value of the parameter $\gamma$. 
Dealing with the post-inflationary evolution, we consider a two-component universe filled by non-interacting radiation and dark matter (we ignore the small contribution from the baryons at the radiation-dominated epoch). Using the basic system of equations (6.16), (6.17) and (6.23)-(6.28), it is possible to derive a closed system of second-order differential equations for the two convenient variables, namely, the potential $\Phi$ and the entropy perturbation

$$
s_{m}:=\frac{3}{4} \delta_{r}-\delta_{m},
$$

where, in this subsection, we have made the notation $\delta_{r}=\delta \rho_{r} / \rho_{r}$ and $\delta_{m}=\delta \rho_{m} / \rho_{m}$ for radiation and dark matter, respectively. One of these two equations is just equation (6.25), and the combined system in Fourier space reads [equation (8.3) is derived in the appendix]

$$
\begin{aligned}
& \Phi^{\prime \prime}+3 \mathcal{H}\left(1+c_{s}^{2}\right) \Phi^{\prime}+\left[k^{2} c_{\mathrm{eff}}^{2}+2 \mathcal{H}^{\prime}+\mathcal{H}^{2}\left(1+3 c_{s}^{2}\right)\right] \Phi=4 \pi G a^{2} c_{s}^{2} \rho_{m} s_{m} \\
& \frac{1}{3 c_{s}^{2}} s_{m}^{\prime \prime}+\mathcal{H} s_{m}^{\prime}+\frac{k^{2}}{4} \frac{\rho_{m}}{\rho_{r}} s_{m}=\frac{k^{4}}{16 \pi G a^{2} \rho_{r}} \Phi
\end{aligned}
$$

System (8.2), (8.3) differs from the corresponding system in general relativity only by the presence of $c_{\text {eff }}^{2}$ instead of $c_{s}^{2}$ in one place in (8.2), where $c_{s}^{2}$ and $c_{\text {eff }}^{2}$ are respectively given by

$$
c_{s}^{2}=\frac{1}{3}\left(1+\frac{3 \rho_{m}}{4 \rho_{r}}\right)^{-1}, \quad c_{\mathrm{eff}}^{2}=c_{s}^{2}+\frac{2(\rho+p)}{3 \kappa+4 \rho} .
$$

Once a solution to (8.2) and (8.3) is found, the density contrasts can be determined using (8.1) and (6.26):

$$
\begin{aligned}
\delta_{r} & =-\frac{3 c_{s}^{2}}{4 \pi G a^{2} \rho_{r}}\left[3 \mathcal{H} \Phi^{\prime}+\left(k^{2}+3 \mathcal{H}^{2}\right) \Phi\right]+\frac{4 \mathcal{H}}{a^{2}} \chi^{\prime}+3 c_{s}^{2} \frac{\rho_{m}}{\rho_{r}} s_{m}, \\
\delta_{m} & =-\frac{9 c_{s}^{2}}{16 \pi G a^{2} \rho_{r}}\left[3 \mathcal{H} \Phi^{\prime}+\left(k^{2}+3 \mathcal{H}^{2}\right) \Phi\right]+\frac{3 \mathcal{H}}{a^{2}} \chi^{\prime}-3 c_{s}^{2} s_{m},
\end{aligned}
$$

where $\chi$ is expressed through $\Phi$ by (6.23). These equations formally differ from their general-relativistic counterparts only by the terms containing $\chi$.

At the radiation-dominated epoch, equations (8.2) and (8.3) take the simple form

$$
\begin{aligned}
\eta^{2} \Phi^{\prime \prime}+4 \eta \Phi^{\prime}+k^{2} c_{\mathrm{eff}}^{2} \eta^{2} \Phi & =\frac{1}{2} \Upsilon \eta s_{m}, \\
\eta^{2} s_{m}^{\prime \prime}+\eta s_{m}^{\prime}+\frac{k^{2}}{4} \Upsilon \eta^{3} s_{m} & =\frac{k^{4}}{6} \eta^{4} \Phi,
\end{aligned}
$$

respectively. Here $\Upsilon$ is a dimensionless parameter defined by

$$
\Upsilon=\left(\frac{8 \pi G}{3} \rho_{\mathrm{eq}} a_{\mathrm{eq}}^{2}\right)^{1 / 2}, \quad \Upsilon \eta \ll 1,
$$

and $a_{\text {eq }}$ and $\rho_{\text {eq }}$ are the scale factor and radiation density at the radiation-matter equality: $\rho_{m}=\rho_{r}=\rho_{\text {eq }}$.

If the parameter $\gamma$ is such that the equality $\rho=\gamma \rho_{\Lambda}$ is reached during inflation, then the evolution of the spectrum at the radiation-dominated stage is the same as in general 
relativity. This follows from equations (8.2) and (8.3), which, in this case, coincide with those of general relativity at the radiation-dominated stage. Since inflation typically ends at energy densities $\rho_{\text {inf }} \sim 10^{-12} / 4 \pi G^{2}$, and since

$$
\gamma \rho_{\Lambda} \approx \frac{1}{8 \pi G \ell^{2}}, \quad \gamma \gg 1
$$

we have the estimate for $\ell$ in this case:

$$
\ell<10^{6} \ell_{P} \sim 10^{-27} \mathrm{~cm}
$$

where $\ell_{P}=G^{1 / 2} \simeq 10^{-33} \mathrm{~cm}$ is the Planck length. Such small values of $\ell$ make the theory practically indistinguishable from the general relativity (which, we remember, is obtained in the limit $\ell \rightarrow 0$ ).

Assume that $\gamma \gg 1$ is such that the condition $\rho=\gamma \rho_{\Lambda}$ takes place at the radiationdominated epoch. Let us determine the range of values of the parameter $\ell$ for which this is the case. The energy density $\rho$ at the matter-radiation dominated stage is expressed as

$$
\rho \simeq \frac{3 H_{0}^{2}}{8 \pi G}\left[\Omega_{m}(1+z)^{3}+\Omega_{r}(1+z)^{4}\right]=\frac{3 H_{0}^{2}}{8 \pi G} \Omega_{m}(1+z)^{3}\left[1+\frac{1+z}{1+z_{\mathrm{eq}}}\right],
$$

where $z_{\mathrm{eq}}$ is the redshift of matter-radiation equality, $H_{0}$ is the current value of the Hubble parameter, and $\Omega_{r}$ and $\Omega_{m}$ are the radiation and matter density parameters, respectively. When making numerical estimates, for definiteness, we will use the recently determined values of these parameters [12]:

$$
\Omega_{m} h_{75}^{2} \approx 0.24, \quad z_{\mathrm{eq}} \simeq 3 \times 10^{3},
$$

where $h_{75}=H_{0} / 75 \mathrm{~km} / \mathrm{s} \mathrm{Mpc} \approx 1$.

The condition $\gamma \rho_{\Lambda}=\rho$ defines the redshift $z_{\ell}$ :

$$
1+z_{\ell}=\left(\frac{1+z_{\mathrm{eq}}}{3 H_{0}^{2} \Omega_{m} \ell^{2}}\right)^{1 / 4}, \quad z_{\ell} \gg z_{\mathrm{eq}},
$$

and the condition $z_{\ell}>z_{\text {eq }}$ implies

$$
\ell<\left[6 \Omega_{m} H_{0}^{2}\left(1+z_{\mathrm{eq}}\right)^{3}\right]^{-1 / 2} \simeq 20 \mathrm{kpc} .
$$

For the modes that enter the Hubble radius well in the GR regime, i.e., at $z<z_{\ell}$, the spectrum of perturbations will not be modified by the end of the radiation-dominated stage as compared to general relativistic cosmology, which can be seen as follows. Before the Hubble-radius crossing, the terms with $k$ in system $(8.2)$ and (8.3) can be dropped, after which this system coincides with the corresponding system in GR. After the transition to GR regime, when $c_{\text {eff }}^{2}$ becomes equal to $c_{s}^{2}$, the system again coincides with that of general relativity for all scales $k$, as was already discussed in Sec. 7.3. Therefore, the behavior of the solutions $\Phi$ and $s_{m}$ is general-relativistic during the whole evolution. The non-standard terms with $\chi$ in equations (8.5) and (8.6) also become unimportant in the GR regime. Hence, the density contrasts are expressed through the basic functions $\Phi$ and $s_{m}$ also in 
a general-relativistic way. Therefore, their amplitudes and phases will not be modified compared to the general-relativistic expressions.

The boundary value of the comoving spatial scale $\lambda_{\ell}=a_{0} / k_{\ell}$ that we were talking about is given by the condition that the corresponding wave crosses the Hubble radius at redshift $z_{\ell}$. We have

$$
k_{\ell}=\mathcal{H}_{\ell}=\frac{a_{\ell}}{\sqrt{3} \ell},
$$

from which, using (8.14), we get

$$
\lambda_{\ell}=\frac{a_{0}}{k_{\ell}}=\sqrt{3}\left(1+z_{\ell}\right) \ell \simeq \lambda_{\mathrm{eq}}\left(\frac{1+z_{\ell}}{1+z_{\mathrm{eq}}}\right)\left(\frac{\ell}{20 \mathrm{kpc}}\right) \simeq 100\left(\frac{\ell}{20 \mathrm{kpc}}\right)^{1 / 2} \mathrm{Mpc}
$$

where $\lambda_{\mathrm{eq}}=a_{0} / k_{\mathrm{eq}} \simeq 100 \mathrm{Mpc}$ is the comoving spatial scale corresponding to Hubbleradius crossing at matter-radiation equality, and, we remember, this estimate works for $\ell<20 \mathrm{kpc}$. For $\lambda>\lambda_{\ell}$, or $k<k_{\ell}=a_{0} / \lambda_{\ell}$, the spectrum of perturbations will not be modified by the end of the radiation-dominated stage.

Consider now the modes that enter the Hubble radius at the modified-gravity epoch, where $c_{\text {eff }}^{2} \approx 1$. Such modes satisfy $k \gg k_{\ell}$; their comoving spatial scales, therefore, are considerably smaller than (8.17). In studying the evolution of adiabatic perturbations on such scales, for not very large values of $\eta$, we can neglect the terms with $\Upsilon$ in equations (8.7) and (8.8). Then, before the transition to the GR regime, we have $c_{\text {eff }}^{2} \approx$ const $=1$, and the exact solution of this system that describes the non-decaying mode with the property $s_{m} \rightarrow 0$ as $\eta \rightarrow 0$ is given by

$$
\begin{aligned}
\Phi & =\frac{3 \Phi_{0}}{\left(k c_{\mathrm{eff}} \eta\right)^{2}}\left(\frac{\sin k c_{\mathrm{eff}} \eta}{k c_{\mathrm{eff}} \eta}-\cos k c_{\mathrm{eff}} \eta\right), \\
s_{m} & =\frac{\Phi_{0}}{c_{\mathrm{eff}}^{4}}\left[\frac{\cos k c_{\mathrm{eff}} \eta-1}{2}-\int_{0}^{k c_{\mathrm{eff}} \eta} \frac{\cos x-1}{x} d x\right] \\
& =\frac{\Phi_{0}}{c_{\mathrm{eff}}^{4}}\left[\frac{\cos k c_{\mathrm{eff}} \eta-1}{2}+\mathcal{C}+\ln k c_{\mathrm{eff}} \eta-\operatorname{Ci}\left(k c_{\mathrm{eff}} \eta\right)\right],
\end{aligned}
$$

where $\Phi \approx \Phi_{0}=$ const at $k \eta \ll 1, \mathcal{C} \approx 0.577$ is the Euler constant, and $\mathrm{Ci}(x)$ is the cosine integral. We have retained the constant $c_{\text {eff }}$ in equations (8.18) and (8.19) which, therefore, will be applicable also to the case of general relativity, where $c_{\text {eff }}^{2}=c_{s}^{2}=1 / 3$.

Consider now a wave with a fixed comoving wave number $k$. It enters the Hubble radius at the moment $\eta \sim 1 / c_{\text {eff }} k$. Thus, when we are in the modified gravity regime the moment of Hubble-radius crossing occurs for a given wave earlier than would be the case in GR. The oscillations in $\Phi$ thus start earlier, and the amplitude of the gravitational potential $\Phi$ also drops more than in GR. A similar suppression effect is also present in the matter power spectrum. From the above expression for the entropy perturbation we learn that $s_{m} \propto(k \eta)^{4}$ for $k c_{\mathrm{eff}} \eta \ll 1$. Thus, the entropy perturbation grows till the mode enters the Hubble radius where oscillations start. Since in the modified gravity epoch the Hubble-radius entry occurs earlier, the entropy perturbation grows considerably less then would be the case in GR and a suppression of matter power spectrum ensues. These effects can be estimated as follows. 
Well after the Hubble-radius crossing (at $k c_{e f f} \eta>1$ ), we can proceed to a new function $u=\eta^{2} \Phi$, for which equation 8.7 with zero right-hand side gives

$$
u^{\prime \prime}+\left(k^{2} c_{\mathrm{eff}}^{2}-\frac{2}{\eta^{2}}\right) u=0 .
$$

Therefore, an approximate solution to $\Phi$ in the regime $k^{2} c_{\text {eff }}^{2} \gg \mathcal{H}^{2}=\eta^{-2}$ can be given in the WKB form

$$
\Phi \propto \frac{1}{\eta^{2} \sqrt{c_{\mathrm{eff}}}} \cos \int k c_{\mathrm{eff}} d \eta .
$$

Comparing this with the leading terms in (8.18), (8.19), we conclude that the WKB solution well after the Hubble-radius crossing can be approximated by

$$
\begin{aligned}
\Phi & \approx-\frac{3 \Phi_{0}}{\left(k c_{\mathrm{eff}}^{0} \eta\right)^{2}} \sqrt{\frac{c_{\mathrm{eff}}^{0}}{c_{\mathrm{eff}}}} \cos \int_{0}^{\eta} k c_{\mathrm{eff}}\left(\eta^{\prime}\right) d \eta^{\prime}, \\
s_{m} & \approx \frac{\Phi_{0}}{2\left(c_{\mathrm{eff}}^{0}\right)^{2} c_{\mathrm{eff}}^{2}} \sqrt{\frac{c_{\mathrm{eff}}^{0}}{c_{\mathrm{eff}}}} \cos \int_{0}^{\eta} k c_{\mathrm{eff}}\left(\eta^{\prime}\right) d \eta^{\prime}+\frac{\Phi_{0}}{\left(c_{\mathrm{eff}}^{0}\right)^{4}}\left(\mathcal{C}-\frac{1}{2}+\ln k c_{\mathrm{eff}}^{0} \eta\right),
\end{aligned}
$$

where $c_{\mathrm{eff}}^{0}$ is the asymptotic initial value of the effective sound velocity, which we have inserted here for comparison with the case of general relativity. The second term in (8.23) is just a source-free solution of equation (8.8); therefore, we have retained its form without any modification.

We note that the same expressions (8.22), 8.23) will be obtained in general relativity with the substitution of $c_{s}$ for $c_{\text {eff }}$ everywhere. Therefore, after the transition from the modified-gravity regime, where $c_{\text {eff }}^{2}(\eta) \approx 1$, to the GR regime, where $c_{\text {eff }}^{2}(\eta)=c_{s}^{2}(\eta) \approx 1 / 3$, solution (8.22) for the potential $\Phi$ will evolve just like in the case of general relativity except that its phase will be shifted and the amplitude of its oscillations will be lower by a factor of

$$
c_{s}^{-3 / 2}=3^{3 / 4} \approx 2.3
$$

This comprises the suppression by $c_{s}^{2}$ due to an earlier entry into the regime of acoustic oscillations with decaying amplitude, as well as amplification of the amplitude by the factor $c_{s}^{-1 / 2}$ that occurs in the transition from the modified-gravity to the GR regime.

The amplitudes of the radiation and matter energy densities on small scales $\left(k c_{\text {eff }} \gg \mathcal{H}\right)$ will be given, according to (8.5), (8.6), by the expressions

$$
\begin{aligned}
& \delta_{r} \approx-\frac{3 c_{s}^{2} k^{2}}{4 \pi G a^{2} \rho_{r}} \Phi=\frac{6 \Phi_{0} c_{s}^{2}}{\left(c_{\mathrm{eff}}^{0}\right)^{2}} \sqrt{\frac{c_{\mathrm{eff}}^{0}}{c_{\mathrm{eff}}}} \cos \int_{0}^{\eta} k c_{\mathrm{eff}}\left(\eta^{\prime}\right) d \eta^{\prime} \\
& \delta_{m} \approx \frac{3}{4} \delta_{r}-3 c_{s}^{2} s_{m} \approx \frac{9 \Phi_{0} c_{s}^{2}}{2\left(c_{\mathrm{eff}}^{0}\right)^{2}} \sqrt{\frac{c_{\mathrm{eff}}^{0}}{c_{\mathrm{eff}}}}\left(1-\frac{1}{3 c_{\mathrm{eff}}^{2}}\right) \cos \int_{0}^{\eta} k c_{\mathrm{eff}}\left(\eta^{\prime}\right) d \eta^{\prime} \\
& -\frac{3 c_{s}^{2} \Phi_{0}}{\left(c_{\mathrm{eff}}^{0}\right)^{4}}\left(\mathcal{C}-\frac{1}{2}+\ln k c_{\mathrm{eff}}^{0} \eta\right) \text {. }
\end{aligned}
$$


We see that the amplitude of $\delta_{r}$, compared with its general-relativistic counterpart, after transition to GR regime (where $c_{\text {eff }}^{2}=c_{s}^{2}=1 / 3$ ), will be lower by the same factor (8.24), which will affect the CMB pattern on small angular scales. If one wishes to exclude this modification in the CMB spectrum at multipoles $l \lesssim 2500$, corresponding to the values reached by ACBAR [13], then one needs to impose the following constraint on the parameter $\ell$ :

$$
\frac{l_{\text {rec }}}{l_{\ell}}=\frac{\lambda_{\ell}}{\lambda_{\text {rec }}}=\frac{\lambda_{\ell}}{\lambda_{\text {eq }}} \frac{\lambda_{\text {eq }}}{\lambda_{\text {rec }}} \simeq 0.4\left(\frac{\ell}{20 \mathrm{kpc}}\right)^{1 / 2} \lesssim \frac{200}{2500} \Rightarrow \ell \lesssim 1 \mathrm{kpc} .
$$

Here, $l_{\text {rec }} \approx 200$ is the characteristic multipole number corresponding to the Hubble radius crossing at recombination, and $\lambda_{\text {rec }}=a_{0} / k_{\text {rec }} \approx 250 \mathrm{Mpc}$ is the corresponding comoving length.

The amplitude of matter perturbation on small scales under consideration is given by (8.26). In general relativity, we would always have $c_{\text {eff }}^{2}=c_{s}^{2}=1 / 3$, and the oscillatory term would always be absent. In the present case of modified gravity, the matter density contrast, apart from the usual monotonic evolution described by the last term in (8.26), also exhibits oscillations. However, these oscillations cease after transition to the GR regime (where $c_{\text {eff }}^{2}=c_{s}^{2}=1 / 3$ ), and the matter density contrast is then described by the usual monotonic term. Its amplitude is smaller than that of general-relativistic expression by the factor $c_{s}^{4}=1 / 9$, although the argument in the logarithm is larger by a factor of $\sqrt{3}$. We thus conclude that, in the eventual power spectrum $P(k) \propto\left|\delta_{m}(k)\right|^{2}$ of dark matter, there will be an additional suppression factor on small scales generated during the radiation-dominated stage:

$$
\frac{P_{\mathrm{mod}}(k)}{P_{\Lambda \mathrm{CDM}}(k)}=\left[\frac{\mathcal{C}-\frac{1}{2}+\ln k \eta_{\mathrm{eq}}}{9\left(\mathcal{C}-\frac{1}{2}+\ln \frac{k \eta_{\mathrm{eq}}}{\sqrt{3}}\right)}\right]^{2} \approx \frac{1}{80}, \quad k \gg k_{\ell}>k_{\mathrm{eq}},
$$

where $P_{\text {mod }}(k)$ and $P_{\Lambda \mathrm{CDM}}(k)$ are the power spectra in the modified gravity theory under consideration and in general relativity, respectively, $\eta_{\mathrm{eq}}$ is the conformal time at the matterradiation equality, and $k_{\ell}$ and its corresponding comoving length are given, respectively, by (8.16) and (8.17). Such a strong suppression will place additional constraint on the value of $\ell$. In order that it does not disturb the observed power above the comoving spatial scales of $\sim 1 \mathrm{Mpc}$, one would require $\lambda_{\ell} \lesssim 1 \mathrm{Mpc}$, or

$$
\ell \lesssim 2 \mathrm{pc}
$$

On the other hand, suppression of the power of linear perturbations on comoving scales below $1 \mathrm{Mpc}$ may be interesting from the viewpoint of the missing-satellite problem (see [14).

During matter domination, there will be an additional modification of power caused by oscillations on scales $k>k_{m}=\mathcal{H} / c_{\text {eff }} \simeq a / \sqrt{2} \ell$ with slightly increasing amplitude $\delta_{m} \propto a^{1 / 4}$ (see Sec. 7.4). The corresponding comoving length scale is

$$
\lambda_{m}=\frac{a_{0}}{k_{m}} \simeq \sqrt{2} \ell(1+z) \simeq 8\left(\frac{1+z}{1+z_{\mathrm{eq}}}\right)\left(\frac{\ell}{2 \mathrm{pc}}\right) \mathrm{kpc} .
$$


At present, the evolution of perturbations on such small spatial scales is non-linear, and the linear-theory analysis of this paper is no longer applicable. On the other hand, it is not easy to probe the spectrum on such scales in the linear regime in the early universe. It is thus hard to see whether the new effect of matter density oscillations could be detected with the currently available data.

The analysis made in this section, in particular, suggests that, if the condition $\rho=$ $\gamma \rho_{\Lambda}$ took place at the matter-dominated stage, which requires values of $\ell$ larger than those given in (8.15), this would modify the power spectra of dark matter and radiation very significantly compared to the case of general relativity, which is important from the viewpoint of current observations. For instance, the pattern in the CMB power spectrum

would be affected because of the essential difference in the effective speed of sound $c_{\text {eff }}^{2}$ from the usual one $c_{s}^{2}$ in this case, and the matter power spectrum would be suppressed on all scales because of specific oscillations on the matter-dominated stage. Whether it is possible to fit all available observations in this case by varying cosmological parameters requires additional investigation using numerical methods.

Finally, we note that we have studied here only the adiabatic mode, for which $s_{m} \rightarrow 0$ asymptotically in the past. For the bound (8.29) on the parameter $\ell$ that arose in this section, the entropy mode is practically excluded, just as it is the case in the $\Lambda$ CDM model. It also remains to be seen whether one can loosen the restrictions on the parameter $\ell$ in the presence of an entropy mode.

\section{Discussion}

The modified theory of gravity that was studied in this paper has several specific features which make it an interesting object of investigation. Expressed in terms of the self-dual twoforms of the Plebański formalism, the theory is local and has the same number of degrees of freedom as general relativity. Due to this property, the theory, in particular, respects the analog of the Birkhoff theorem, possessing a unique one-parameter family of (modified!) spherically symmetric vacuum solutions 断, which also turn out to be static. Unlike some other modifications of general relativity, such as massive gravity, it has a continuous limit to general relativity and, therefore, may be regarded as a smooth deformation of the latter. At the linearized level, the modification is described by a parameter $\ell$ with dimension of length and a dimensionless parameter $g$; they enter the fundamental potentials $V$ and $R$ defined in (2.10) and (2.12), respectively. The value of $g$ is restricted to lie between zero and unity from the requirement of absence of singularity in the equations for linear perturbations, while the parameter $\ell$ is essentially free. The limit of general relativity is obtained as $\ell \rightarrow 0$, irrespective of the value of $g$.

The cosmological properties of the theory also reflect the absence of new degrees of freedom. Thus, the dynamics of an ideally homogeneous and isotropic universe described by the modified gravity under consideration is absolutely the same as in general relativity. For a realistic universe, which is homogeneous only statistically, there will be the problem of averaging which might lead to modification of the effective Friedmann equations, the issue that lies beyond the scope of the present paper. 
The theory of linear perturbations is modified in a very interesting way. Concerning perturbations of the scalar type, first of all, there exists an analog of the relativistic potential $\Phi$ in this theory, which is related to the matter perturbations in the usual way through Eq. (6.24). Just as in general relativity, it is also possible to obtain a system of second-order partial differential equations describing the evolution of this potential; see Eqs. (6.23)-(6.28). This system is different from its counterpart in general relativity. Its analysis, performed in Secs. 7 and 8, reveals the following basic qualitative features:

1. The inflationary theory of generation of primordial perturbation remains intact because corrections due to modification of gravity at the inflationary stage are suppressed by the small ratio $|\dot{H}| / H^{2} \ll 1$.

2. The evolution of perturbations at a radiation-dominated stage, described by (7.34), proceeds with a modified effective time-dependent speed of sound $c_{\text {eff }}$, given by (7.35). The relation between the energy density and relativistic potential, Eq. (7.36), is also modified as compared to general relativity. For sufficiently low values of the length parameter, $\ell<20 \mathrm{kpc}$, given by (8.15), the transition from the modified-gravity regime (where $c_{\text {eff }} \approx 1$ ) to the general-relativistic regime (where $c_{\text {eff }} \approx c_{s} \approx 1 / \sqrt{3}$ ) takes place at the radiation-dominated stage. The modes that enter the Hubble radius after this transition evolve just as in general relativity, so that the spectrum in this long-wave region is not modified. The modes that enter the Hubble radius before this transition experience some suppression in the course of the transition; their amplitude drops by approximately 2.3, see Eq. (8.24). This will affect the CMB angular power spectrum on sufficiently small scales; in order that this spectrum remain unmodified below the multipole number $l \simeq 2500$ reached by ACBAR [13], the fundamental length parameter of the theory is further restricted to be $\ell \lesssim 1 \mathrm{kpc}$; see Eq. (8.27).

Modification of gravity during the radiation-dominated stage also has interesting effect on the evolution of dark-matter perturbations on small scales, which is described in Sec. 8. Specifically, the matter density contrast, apart from the usual monotonic evolution also exhibits oscillations, described by the first term in (8.26), that cease after transition to the GR regime (where $c_{\text {eff }}^{2}=c_{s}^{2}=1 / 3$ ). Moreover, the amplitude of the monotonically growing part of matter perturbation is smaller than the similar expression in general relativity by the factor $c_{s}^{4}=1 / 9$. This leads to a suppression of the eventual power spectrum of dark matter by a factor $\sim 1 / 80$ [see (8.28)] on the scales that enter the Hubble radius well before the transition to general-relativistic regime. In order that this suppression does not disturb the observed power above the comoving spatial scales of $\sim 1 \mathrm{Mpc}$, one would require $\ell \lesssim 2 \mathrm{pc}$, which turns out to be the strongest constraint on this parameter in the present scenario. On the other hand, suppression of the power of linear perturbations on comoving scales below $1 \mathrm{Mpc}$ may be interesting from the viewpoint of the missing-satellite problem (see [14]).

3. The evolution at the matter-dominated stage is qualitatively different from that of general relativity because equation (7.41) for the relativistic potential still contains a 
nonzero effective speed of sound, given by (7.42) or (7.52). For this reason, the closed equation (7.51) for density contrast exhibits oscillatory character on sufficiently small scales; the corresponding comoving spatial scale is given by (8.30). In view of the previous constraint $\ell \lesssim 2 \mathrm{pc}$, this behavior will only affect the comoving spatial scales smaller than about $8 \mathrm{kpc}$. Note, however, that, at present, the evolution of perturbations on such small spatial scales is non-linear, and the linear-theory analysis of this paper is no longer applicable. On the other hand, it is not easy to probe the spectrum on such scales in the linear regime in the early universe. It is thus hard to see whether the new effect of matter density oscillations could be detected with the currently available data.

We should note that the above description is of qualitative character and, therefore, needs to be substantiated by numerical integration of the derived system of exact equations for perturbations, which will be the subject of subsequent work. We also leave to the future work an analysis of the effect of modification on the gravitational waves, with a preliminary description given in Appendix B.

\section{Acknowledgments}

K. K. was supported by an EPSRC Advanced Fellowship. Yu.S. was supported by the "Cosmomicrophysics" programme and Program of Fundamental Research of the Physics and Astronomy Division of the National Academy of Sciences of Ukraine, and by the State Foundation for Fundamental Research of Ukraine under grant F28.2/083.

\section{Appendix}

\section{A. Derivation of equations describing the radiation-dark-matter system}

The conservation equations (6.16) and 6.17), written in Fourier space for a system of matter and radiation with vanishing shear, read

$$
\begin{aligned}
\delta_{m}^{\prime}=\theta_{m}+3 \Psi_{\mathrm{GR}}^{\prime}, & \delta_{r}^{\prime} & =\frac{4}{3} \theta_{r}+4 \Psi_{\mathrm{GR}}^{\prime}, \\
\theta_{m}^{\prime}=-\mathcal{H} \theta_{m}-k^{2} \Phi_{\mathrm{GR}}, & \theta_{r}^{\prime} & =-k^{2} \Phi_{\mathrm{GR}}-\frac{1}{4} k^{2} \delta_{r} .
\end{aligned}
$$

Here, the variable $\theta_{a}$ for each component is defined as

$$
\theta_{a}=-\frac{k^{2} \delta u_{a}}{\rho_{a}+p_{a}}
$$

Equations (6.26) and (6.27) in the case of vanishing shear can be expressed, respectively, as

$$
\begin{aligned}
4 \pi G a^{2}\left(\rho_{m} \delta_{m}+\rho_{r} \delta_{r}\right) & =-3 \mathcal{H}\left(\Phi^{\prime}+\mathcal{H} \Phi\right)-k^{2} \Phi-4 \pi G \rho^{\prime} \chi^{\prime}, \\
4 \pi G a^{2}\left(\rho_{m} \theta_{m}+\frac{4}{3} \rho_{r} \theta_{r}\right) & =-k^{2}\left[\Phi^{\prime}+\mathcal{H} \Phi-4 \pi G(\rho+p) \chi^{\prime}\right] .
\end{aligned}
$$


Differentiating the entropy perturbation $s_{m}=\frac{3}{4} \delta_{r}-\delta_{m}$, using (A.1) and A.2. , we get

$$
s_{m}^{\prime}=\theta_{r}-\theta_{m}, \quad s_{m}^{\prime \prime}=\mathcal{H} \theta_{m}-\frac{k^{2}}{4} \delta_{r}
$$

It remains to calculate the left-hand side of (8.3). Using the definition (8.4) of $c_{s}^{2}$, we have

$$
\begin{aligned}
& \frac{1}{3 c_{s}^{2}} s_{m}^{\prime \prime}+\mathcal{H} s_{m}^{\prime}+\frac{k^{2}}{4} \frac{\rho_{m}}{\rho_{r}} s_{m}= \\
= & \left(1+\frac{3 \rho_{m}}{4 \rho_{r}}\right)\left(\mathcal{H} \theta_{m}-\frac{k^{2}}{4} \delta_{r}\right)+\mathcal{H}\left(\theta_{r}-\theta_{m}\right)+\frac{k^{2}}{4} \frac{\rho_{m}}{\rho_{r}}\left(\frac{3}{4} \delta_{r}-\delta_{m}\right) \\
= & \frac{3 \mathcal{H}}{4 \rho_{r}}\left(\rho_{m} \theta_{m}+\frac{4}{3} \rho_{r} \theta_{r}\right)-\frac{k^{2}}{4 \rho_{r}}\left(\rho_{m} \delta_{m}+\rho_{r} \delta_{r}\right) \\
= & -\frac{3 \mathcal{H}}{4 \rho_{r}} \frac{k^{2}}{4 \pi G a^{2}}\left[\Phi^{\prime}+\mathcal{H} \Phi-4 \pi G(\rho+p) \chi^{\prime}\right] \\
& +\frac{k^{2}}{4 \rho_{r}} \frac{1}{4 \pi G a^{2}}\left[3 \mathcal{H}\left(\Phi^{\prime}+\mathcal{H} \Phi\right)+k^{2} \Phi+4 \pi G \rho^{\prime} \chi^{\prime}\right]=\frac{k^{4}}{16 \pi G a^{2} \rho_{r}} \Phi .
\end{aligned}
$$

Here, in the third equality, we have used equations (A.4) and (A.5), and, in the last equality, the conservation equation $\rho^{\prime}=-3 \mathcal{H}(\rho+p)$. Equation (A.7) is the desired result (8.3).

\section{B. Tensor sector}

\section{B.1 Curvatures}

To describe the linearized connections and the curvature in the tensor sector it is very convenient to introduce the following two differential operators on the space of symmetric traceless transverse matrices:

$$
D X^{i j}:=X_{, l}^{i k} \epsilon^{j k l}+\mathrm{i}\left(X^{i j}\right)^{\prime}, \quad \bar{D} X^{i j}:=X_{, l}^{i k} \epsilon^{j k l}-\mathrm{i}\left(X^{i j}\right)^{\prime},
$$

where, as usual, the prime denotes the conformal time derivative. It is easy to see that the operators $D$ and $\bar{D}$ transform symmetric transverse traceless matrices into matrices with similar properties and that

$$
D \bar{D}=\bar{D} D=-\square
$$

where we have denoted $\square:=-\partial_{\eta}^{2}+\Delta$. In terms of the derivative operators introduced, we get

$$
\delta A_{\chi}^{i}=-\frac{1}{a^{3}} D\left(a \chi^{i j}\right) d x^{j}, \quad \delta A_{\rho}^{i}=-\frac{1}{a} \bar{D}\left(a \rho^{i j}\right) d x^{j} .
$$

For a linearized connection of the form $\delta A^{i}=A^{i j} d x^{j}$ with a symmetric traceless matrix $A^{i j}$, the linearized curvature is given by the following expression:

$$
\mathcal{D}_{0} \delta A^{i}=\frac{1}{2 a} \bar{D}\left(\frac{A^{i j}}{a}\right) \Sigma_{0}^{j}-\frac{1}{2 a^{3}} D\left(a A^{i j}\right) \bar{\Sigma}_{0}^{j}
$$


Thus, we have

$$
\begin{aligned}
& \mathcal{D}_{0} \delta A_{\chi}^{i}=-\frac{1}{2 a} \bar{D}\left[\frac{D\left(a \chi^{i j}\right)}{a^{4}}\right] \Sigma_{0}^{j}+\frac{1}{2 a^{3}} \bar{D}\left[\frac{D\left(a \chi^{i j}\right)}{a^{2}}\right] \bar{\Sigma}_{0}^{j}, \\
& \mathcal{D}_{0} \delta A_{\rho}^{i}=-\frac{1}{2 a} \bar{D}\left[\frac{\bar{D}\left(a \rho^{i j}\right)}{a^{2}}\right] \Sigma_{0}^{j}+\frac{1}{2 a^{3}} D \bar{D}\left(a \rho^{i j}\right) \bar{\Sigma}_{0}^{j} .
\end{aligned}
$$

Note that the derivative operators $D$ and $\bar{D}$ do not act on the basis two-forms in these expressions.

\section{B.2 Field equations}

For tensor perturbations, there is no source on the right-hand side of (4.8), and we get the following simple system of equations:

$$
\begin{aligned}
& a \bar{D}\left[\frac{D\left(a \chi^{i j}\right)}{a^{4}}\right]-\frac{1}{a^{2}}\left(\mathcal{H}^{2}+\mathcal{H}^{\prime}\right) \chi^{i j}+a \bar{D}\left[\frac{\bar{D}\left(a \rho^{i j}\right)}{a^{2}}\right]-\left(\mathcal{H}^{2}-\mathcal{H}^{\prime}\right) \rho^{i j}=8 \pi G \kappa \chi^{i j}, \\
& \frac{1}{a} D\left[\frac{D\left(a \chi^{i j}\right)}{a^{2}}\right]-\frac{1}{a^{2}}\left(\mathcal{H}^{2}-\mathcal{H}^{\prime}\right) \chi^{i j}+\frac{1}{a} D \bar{D}\left(a \rho^{i j}\right)-\left(\mathcal{H}^{2}+\mathcal{H}^{\prime}\right) \rho^{i j}=0 .
\end{aligned}
$$

Now expanding the derivative operators one obtains the following coupled system of secondorder differential equations:

$$
\begin{aligned}
\frac{1}{a^{2}}\left(\partial_{\eta}^{2}-\Delta-2 \mathcal{H} \partial_{\eta}-4 \mathcal{H}^{2}-8 \pi G a^{2} \kappa+4 \mathrm{i} \mathcal{H} \epsilon\right) \chi^{i j} & =\left(\partial_{\eta}^{2}+\Delta+2 \mathrm{i} \partial_{\eta} \epsilon\right) \rho^{i j} \\
a^{2}\left(\partial_{\eta}^{2}-\Delta+2 \mathcal{H} \partial_{\eta}\right) \rho^{i j} & =\left(\partial_{\eta}^{2}+\Delta-2 \mathrm{i} \partial_{\eta} \epsilon\right) \chi^{i j}
\end{aligned}
$$

where $\epsilon$ is a first-order differential operator that acts on the space of symmetric trace-free matrices via:

$$
\epsilon X^{i j}=X_{, l}^{i k} \epsilon^{j k l} .
$$

We note that $\epsilon^{2}=-\Delta$. As a check, in the limit $\chi^{i j} \rightarrow 0$ such that $\kappa \chi^{i j}$ is kept finite, we get the GR result $\left(\partial_{\eta}^{2}-\Delta+2 \mathcal{H} \partial_{\eta}\right) \rho^{i j}=0$.

\section{B.3 Action principle and Hamiltonian analysis}

We have written the system of equations $(\overline{B .9}),(B .10)$ in the above form to make it obvious that they can be obtained as Euler-Lagrange equations for the following Lagrangian:

$$
\begin{aligned}
\mathcal{L}_{\text {tens }}= & -\frac{a^{2}}{2} \rho\left(\partial_{\eta}^{2}-\Delta+2 \mathcal{H} \partial_{\eta}\right) \rho+\chi\left(\partial_{\eta}^{2}+\Delta+2 \mathrm{i} \partial_{\eta} \epsilon\right) \rho \\
& -\frac{1}{2 a^{2}} \chi\left(\partial_{\eta}^{2}-\Delta-2 \mathcal{H} \partial_{\eta}-4 \mathcal{H}^{2}-8 \pi G \kappa a^{2}+4 \mathrm{i} \mathcal{H} \epsilon\right) \chi,
\end{aligned}
$$

where we have dropped the internal indices for brevity. Indeed, one easily obtains both equations using the fact that the operators $\partial_{\eta}^{2}-\Delta+2 \mathcal{H} \partial_{\eta}, \partial_{\eta}^{2}$, and $\partial_{\eta}^{2}-\Delta-2 \mathcal{H} \partial_{\eta}$ are self-adjoint with respect to the inner products given by

$$
\langle\phi, \psi\rangle_{+}=\int d \eta d^{3} x a^{2} \phi \psi, \quad\langle\phi, \psi\rangle_{0}=\int d \eta d^{3} x \phi \psi, \quad\langle\phi, \psi\rangle_{-}=\int d \eta d^{3} x \frac{1}{a^{2}} \phi \psi,
$$


respectively. Moreover, the operator $\epsilon$ is self-adjoint with any of these inner products.

It is clear from the form of this Lagrangian that it is degenerate and thus describes just one propagating field, not two. Indeed, the time derivatives of the fields enter in the combination $\left(a^{2} / 2\right)\left(\rho^{\prime}-\chi^{\prime} / a^{2}\right)^{2}$, which makes it clear that the system is degenerate. There are many ways to unravel the dynamics of such a system, but the most powerful, if not always the easiest method is via its Hamiltonian analysis. Thus, let us rewrite it in the Hamiltonian form. To this end, we first rewrite the Lagrangian in the form that makes it obvious what the canonical momenta are. Integrating by parts in the terms containing second time derivatives, we write:

$$
\begin{aligned}
\mathcal{L}_{\text {tens }}= & \frac{a^{2}}{2}\left(\rho^{\prime 2}+\rho \Delta \rho\right)-\left(\epsilon \rho-\mathrm{i} \rho^{\prime}\right)\left(\epsilon \chi+\mathrm{i} \chi^{\prime}\right) \\
& +\frac{1}{2 a^{2}}\left[\chi^{\prime 2}+\chi \Delta \chi-4 \mathrm{i} \mathcal{H} \chi \epsilon \chi+\left(4 \mathcal{H}^{2}+8 \pi G a^{2} \kappa\right) \chi^{2}\right] .
\end{aligned}
$$

Now the conjugate momenta are:

$$
\pi_{\rho}=a^{2} \rho^{\prime}-\chi^{\prime}+\mathrm{i} \epsilon \chi, \quad \pi_{\chi}=\frac{1}{a^{2}} \chi^{\prime}-\rho^{\prime}-\mathrm{i} \epsilon \rho .
$$

Thus, the following primary constraint holds:

$$
\phi_{1}:=\pi_{\rho}+a^{2} \pi_{\chi}+\mathrm{i} \epsilon\left(a^{2} \rho-\chi\right)=0 .
$$

The Hamiltonian is obtained as $\mathcal{H}_{\text {tens }}=\pi_{\rho} \rho^{\prime}+\pi_{\chi} \chi^{\prime}-\mathcal{L}_{\text {tens }}+u \phi_{1}$, where $u$ is a Lagrange multiplier and $\phi$ is the primary constraint. The Hamiltonian then must be written in terms of the momenta. Only one momentum is needed for this, for which we choose $\pi_{\rho}$. We get:

$$
\mathcal{H}_{\text {tens }}=\frac{1}{2 a^{2}} \pi_{\rho}^{2}-\frac{1}{a^{2}} \pi_{\rho} \mathrm{i} \epsilon \chi-\frac{a^{2}}{2} \rho \Delta \rho-\rho \Delta \chi+\frac{1}{2 a^{2}}\left(4 \mathrm{i} \mathcal{H} \chi \epsilon \chi-4 \mathcal{H}^{2} \chi^{2}-8 \pi G a^{2} \kappa \chi^{2}\right)+u \phi_{1} .
$$

As a check, one can verify that the Poisson brackets of this Hamiltonian with the phase space variables $\pi_{\rho}, \pi_{\chi}, \rho$, and $\chi$ give rise precisely to the original equations (B.9), (B.10) with the Lagrange multiplier $u=\chi^{\prime} / a^{2}$ as a consequence of $\chi^{\prime}=\left\{H_{\text {tens }}, \chi\right\}=a^{2} u$.

Now the condition that the constraint $\phi_{1}=0$ is preserved in time gives the secondary constraint:

$$
\phi_{2}:=\phi_{1}^{\prime}=2 a^{2} \mathcal{H}\left(\pi_{\chi}+\mathrm{i} \epsilon \rho\right)+\left\{H_{\text {tens }}, \phi_{1}\right\}=0 .
$$

We find:

$$
\phi_{2}:=a^{2} \Delta \rho+\mathrm{i} \epsilon \pi_{\rho}-\mathcal{H} \pi_{\rho}-\mathrm{i} \mathcal{H} \epsilon \chi+2 \mathcal{H}^{2} \chi+4 \pi G a^{2} \kappa \chi=0,
$$

where we have used the relation $a^{2}\left(\pi_{\chi}+\mathrm{i} \epsilon \rho\right)=\mathrm{i} \epsilon \chi-\pi_{\rho}$ that follows from $\phi_{1}=0$. As a check, we note that $(\overline{B .19})$ is precisely the difference of the two original equations $(\overline{B .9})$, (B.10).

Now one can see that the Poisson bracket of $\phi_{1}$ with $\phi_{2}$ is a constant, and so the constraints are second class. These constraints can be used to solve for $\pi_{\chi}$ and $\chi$ in terms of the phase space variables $\pi_{\rho}$ and $\rho$. After that, one should substitute the result into (B.17) to obtain an effective Hamiltonian with second class constraints solved for. In addition, one also has to compute the arising Dirac bracket. 
To solve (B.19), we introduce:

$$
m^{2}:=2 \mathcal{H}^{2}+4 \pi G a^{2} \kappa
$$

We now get for $\chi$ :

$$
\chi=\left(\mathrm{i} \mathcal{H} \epsilon-m^{2}\right)^{-1}\left[a^{2} \Delta \rho+(\mathrm{i} \epsilon-\mathcal{H}) \pi_{\rho}\right],
$$

where the inverse of $\left(\mathrm{i} \mathcal{H} \epsilon-m^{2}\right)$ should be interpreted by passing to the momentum space. There is no operator-ordering ambiguities in this formula as all the operators only involve spatial derivatives and thus commute.

We now note that Hamiltonian (B.17) can be rewritten on the constraint surface (B.19) as

$$
\mathcal{H}_{\text {tens }}^{\text {eff }}=\frac{1}{2 a^{2}} \pi_{\rho}^{2}-\frac{a^{2}}{2} \rho \Delta \rho-\frac{\mathcal{H}}{a^{2}} \chi\left(\pi_{\rho}-\mathrm{i} \epsilon \chi\right),
$$

where $\chi$ is to be substituted from (B.21). The action in the Hamiltonian form on the constraint surface now reads:

$$
S_{\text {tens }}^{\mathrm{eff}}=\int d \eta d^{3} x\left[\rho^{\prime} \pi_{\rho}+\frac{\chi^{\prime}}{a^{2}} \mathrm{i} \epsilon \chi-\frac{\chi^{\prime}}{a^{2}}\left(\pi_{\rho}+\mathrm{i} a^{2} \epsilon \rho\right)-\mathcal{H}_{\mathrm{tens}}^{\mathrm{eff}}\right],
$$

where we have used the constraint $(\overline{B .16})$ to express $\pi_{\chi}$ in terms of the other phase space variables. Now, the term

$$
\frac{\chi^{\prime}}{a^{2}} \mathrm{i} \epsilon \chi-\frac{\mathcal{H}}{a^{2}} \chi \mathrm{i} \epsilon \chi=\left(\frac{\chi \mathrm{i} \epsilon \chi}{2 a^{2}}\right)^{\prime}
$$

here is a total time derivative and can be dropped. This leaves us with

$$
S_{\text {tens }}^{\text {eff }}=\int d \eta d^{3} x\left[\rho^{\prime} \pi_{\rho}-\frac{\chi^{\prime}}{a^{2}}\left(\pi_{\rho}+\mathrm{i} a^{2} \epsilon \rho\right)-\frac{1}{2 a^{2}} \pi_{\rho}^{2}+\frac{a^{2}}{2} \rho \Delta \rho+\frac{\mathcal{H}}{a^{2}} \chi \pi_{\rho}\right],
$$

where (B.21) must be substituted. After this is done, and all the dust settles, we get the following effective action in the Hamiltonian form:

$$
\begin{aligned}
S_{\text {tens }}^{\mathrm{eff}}= & \int d \eta d^{3} x\left(\rho^{\prime} \frac{m^{2} \pi_{\rho}}{m^{2}-\mathrm{i} \mathcal{H} \epsilon}-\frac{1}{2 a^{2}} \pi_{\rho}^{2}\left[1+\left(\frac{\mathcal{H}-\mathrm{i} \epsilon}{m^{2}-\mathrm{i} \mathcal{H} \epsilon}\right)^{\prime}\right]\right. \\
& \left.+\Delta \rho \frac{\pi_{\rho}}{a}\left(\frac{a}{m^{2}-\mathrm{i} \mathcal{H} \epsilon}\right)^{\prime}+\frac{a^{2}}{2} \rho \Delta \rho\left[1+\frac{\mathrm{i} \epsilon}{a^{2}}\left(\frac{a^{2}}{m^{2}-\mathrm{i} \mathcal{H} \epsilon}\right)^{\prime}\right]\right) .
\end{aligned}
$$

As before, the limit to GR is easily obtained by sending $m^{2} \rightarrow \infty$. Another simple limit is that of passing to the Minkowski spacetime background. This is obtained by setting $\mathcal{H}=0$ and $a=1$ everywhere, as well as taking $m^{2}$ to be time-independent. One again obtains an unmodified system, even for a finite $m^{2}$, which is consistent with the earlier observations in the literature that the gravitational waves in the Minkowski spacetime are unmodified. However, we see that tensor perturbations around an expanding universe are modified quite non-trivially. 
This action can be further rewritten in the usual form depending on generalized velocities only by integrating out the momentum $\pi_{\rho}$. We get

$$
\begin{aligned}
S_{\text {tens }}^{\text {eff }}= & \int d \eta d^{3} x \frac{a^{2}}{2}\left(\left[1+\left(\frac{\mathcal{H}-\mathrm{i} \epsilon}{m^{2}-\mathrm{i} \mathcal{H} \epsilon}\right)^{\prime}\right]^{-1}\left[\rho^{\prime} \frac{m^{2}}{m^{2}-\mathrm{i} \mathcal{H} \epsilon}+\frac{\Delta \rho}{a}\left(\frac{a}{m^{2}-\mathrm{i} \mathcal{H} \epsilon}\right)^{\prime}\right]^{2}\right. \\
& \left.+\rho \Delta \rho\left[1+\frac{\mathrm{i} \epsilon}{a^{2}}\left(\frac{a^{2}}{m^{2}-\mathrm{i} \mathcal{H} \epsilon}\right)^{\prime}\right]\right) .
\end{aligned}
$$

The resulting effective action is complex. The prescription for dealing with such complex actions advocated in [5] was to require the metric to be real and take the real part of the action. However, a different prescription may also be possible. ${ }^{4}$ Thus, it may be that the appropriate prescription is to allow the action to be a complex, holomorphic function of the metric $g_{\mu \nu}$, with the latter treated as a collection of complex variables. Then, the condition that the imaginary part of the action vanishes gives a relation allowing to express the imaginary part of the metric in terms of its real part. The real part of the action can then be written as a functional of the real part of the metric. More work is needed to decide which prescription for dealing with "reality conditions" for this theory is appropriate. We leave an analysis of the tensor sector that depends on this prescription to future research.

An alternative quick way to obtain the field equation for $\rho$ that stems from $(\overline{B .27})$ is as follows. One notes that equation $(\overline{B .10})$ can be written as

$$
\pi_{\rho}^{\prime}-a^{2} \Delta \rho-\mathrm{i} \epsilon\left(\mathrm{i} \epsilon-\partial_{\eta}\right) \chi=0 .
$$

On the other hand, from the definition (B.15) of the momentum $\pi_{\rho}$, it follows that

$$
\left(\mathrm{i} \epsilon-\partial_{\eta}\right) \chi=\pi_{\rho}-a^{2} \rho^{\prime} .
$$

This gives an equation that involves only $\rho$ and $\pi_{\rho}$ :

$$
\pi_{\rho}^{\prime}-a^{2} \rho-\mathrm{i} \epsilon\left(\pi_{\rho}-a^{2} \rho^{\prime}\right)=0 .
$$

One can rewrite this as

$$
\left(\partial_{\eta}-\mathrm{i} \epsilon\right)\left(\pi_{\rho}+\mathrm{i} a^{2} \epsilon \rho\right)-2 \mathcal{H} a^{2} \mathrm{i} \epsilon \rho=0 .
$$

Using the expression for $\pi_{\rho}$ that was obtained above (when integrating it out), or substituting $\chi$ into the definition (B.15) of the momentum $\pi_{\rho}$ and solving for $\pi_{\rho}$, we obtain the following compact expression for the combination

$$
\pi_{\rho}+\mathrm{i} a^{2} \epsilon \rho=a^{2}\left[1+\left(\frac{\mathcal{H}-\mathrm{i} \epsilon}{m^{2}-\mathrm{i} \mathcal{H} \epsilon}\right)^{\prime}\right]^{-1}\left(\partial_{\eta}+\mathrm{i} \epsilon\right) \frac{m^{2} \rho}{m^{2}-\mathrm{i} \mathcal{H} \epsilon} .
$$

As a result, we get the following differential equation for $\rho$ :

$$
\left(\partial_{\eta}-\mathrm{i} \epsilon\right) a^{2}\left[1+\left(\frac{\mathcal{H}-\mathrm{i} \epsilon}{m^{2}-\mathrm{i} \mathcal{H} \epsilon}\right)^{\prime}\right]^{-1}\left(\partial_{\eta}+\mathrm{i} \epsilon\right) \frac{m^{2} \rho}{m^{2}-\mathrm{i} \mathcal{H} \epsilon}-2 \mathcal{H} a^{2} \mathrm{i} \epsilon \rho=0 .
$$

As a check, we note that the resulting equation takes the general-relativistic form $\left(a^{2} \rho^{\prime}\right)^{\prime}-$ $a^{2} \Delta \rho=0$ in the limit $m^{2} \rightarrow \infty$. We shall not analyze the arising modified gravitational wave equations in this paper, leaving this to future work.

\footnotetext{
${ }^{4}$ One of the authors $(\mathrm{KK})$ is grateful to Laurent Freidel for a discussion that led to an alternative prescription.
} 


\section{References}

[1] C. Skordis, Phys. Rev. D 79 (2009) 123527 arXiv:0806.1238.

[2] K. Krasnov, Renormalizable Non-Metric Quantum Gravity?, hep-th/0611182.

[3] I. Bengtsson, Phys. Lett. B 254 (1991) 55.

[4] K. Krasnov and Yu. Shtanov, Class. and Quant. Grav. 25 (2008) 025002 arXiv:0705.2047.

[5] K. Krasnov, Phys. Rev. D 81 (2010) 084026, arXiv:0911.4903.

[6] S. M. Carroll, I. Sawicki, A. Silvestri and M. Trodden, New J. Phys. 8 (2006) 323 astro-ph/0607458.

[7] K. Krasnov, Phys. Rev. D 79 (2009) 044017 arXiv:0812.3603.

[8] K. Krasnov, Plebanski Formulation of General Relativity: A Practical Introduction, arXiv:0904.0423.

[9] K. Krasnov, Phys. Rev. Lett. 100 (2008) 081102 arXiv:0711.0090.

[10] K. Krasnov and Y. Shtanov, Int. J. Mod. Phys. D 17 (2009) 2555 arXiv:0805.2668

[11] V. Mukhanov, Physical Foundations of Cosmology, Cambridge University Press, Cambridge (2005).

[12] E. Komatsu et al., Seven-Year Wilkinson Microwave Anisotropy Probe (WMAP) Observations: Cosmological Interpretation, arXiv:1001.4538.

[13] C. L. Reichardt et al., Astrophys. J. 694 (2009) 1200 arXiv:0801.1491.

[14] A. V. Kravtsov, Dark matter substructure and dwarf galactic satellites, arXiv:0906.3295. 